\title{
DIMENSIONALITY OF LOCAL MINIMIZERS OF THE INTERACTION ENERGY
}

\author{
D. BALAGUÉ ${ }^{1}$, J. A. CARRILlO ${ }^{2}$, T. LAURENT ${ }^{3}$, AND G. RAOUL ${ }^{4}$
}

\begin{abstract}
In this work we consider local minimizers (in the topology of transport distances) of the interaction energy associated to a repulsive-attractive potential. We show how the dimensionality of the support of local minimizers is related to the repulsive strength of the potential at the origin.
\end{abstract}

\section{INTRODUCTION}

Given a Borel measurable function $W: \mathbb{R}^{N} \rightarrow(-\infty,+\infty]$ which is bounded from below, the interaction energy of the Borel probability measure $\mu$ is given by

$$
E_{W}[\mu]:=\frac{1}{2} \iint_{\mathbb{R}^{N} \times \mathbb{R}^{N}} W(x-y) d \mu(x) d \mu(y) .
$$

Our main goal will be to analyse the qualitative properties of local minimizers of the energy $E_{W}$ in the set of Borel probability measures with the topology induced by transport distances. More specifically, we will show that the Hausdorff dimension of the support of local minimizers is directly related to the behavior at the origin of $\Delta W$.

The interaction energy $E_{W}$ arises in many contexts. In physical, biological, and material sciences it is used to model particles or individuals effects on others via pairwise interactions. Given $n$ particles located at $X_{1}, \ldots, X_{n} \in \mathbb{R}^{N}$, their discrete interaction energy is given by

$$
E_{W}^{n}\left[X_{1}, \ldots, X_{n}\right]:=\frac{1}{2 n^{2}} \sum_{\substack{i, j=1 \\ j \neq i}}^{n} W\left(X_{i}-X_{j}\right) .
$$

Note that formally for a large number of particles, the discrete energy (2) is well approximated by the continuum energy (1) where $d \mu(x)$ is a general distribution of particles at location $x \in \mathbb{R}^{N}$. In fact, the continuum energy (1) of the discrete distribution $\frac{1}{n} \sum_{i=1}^{n} \delta_{X_{i}}$ reduces to (2).

In models arising in material sciences [15, 37, 31, 21, 35], particles, nano-particles, or molecules self-assemble in a way to minimize energies similar to $E_{W}^{n}$. Analogously in applications to biological sciences [27, 26, 33, 4, 14], individuals in a social aggregate (e.g., swarm, flock, school, or herd) self-organize in order to minimize similar type of energies. In these applications the potential $W$ is typically repulsive in the short range so that particles/individuals do not collide, and attractive in the long range so that the particles/individuals gather to form a group or a structure. Therefore one is often led to consider radially symmetric interaction potentials of the form $W(x)=w(|x|)$ where $w:[0,+\infty) \rightarrow(-\infty,+\infty]$ is decreasing on some interval $\left[0, r_{0}\right)$ and increasing on $\left(r_{0},+\infty\right)$. The function $w$ may or may not have a singularity at $r=0$. We will refer to such potentials as being repulsive-attractive. Since $w$ has a global minimum at $r_{0}$, it is obvious that if we consider only two particles $X_{1}$ and $X_{2}$, in order to minimize $E_{W}^{2}\left[X_{1}, X_{2}\right]$, the two particles must be located at a distance $r_{0}$ from one another. Whereas the situation is simple with two particles, it becomes very complicated for large number of particles. Recent works [23, 36, 19, 35, 22, 32, 30, 17, 18, 3] have shown that such repulsive-attractive potentials lead to the emergence of surprisingly rich 
geometric structures. The goal of the present paper is to understand how the dimensionality of these structures depends on the singularity of $\Delta W$ at the origin.

Let us describe the main results. Consider a repulsive-attractive potential $W(x)=w(|x|)$. Typically the Laplacian of such potential will be negative in a neighborhood of the origin. We show that if

$$
\Delta W(x) \sim-\frac{1}{|x|^{\beta}} \quad \text { as } x \rightarrow 0
$$

for some $0<\beta<N$, then the support of local minimizers of $E_{W}$ has Hausdorff dimension greater or equal to $\beta$. The precise hypotheses needed on $W$ for this result to be true, as well as the precise meaning of (3), can be found in the statement of Theorem 1 . The exponent $\beta$ appearing in (3) quantifies how repulsive the potential is at the origin. Therefore our result can be intuitively understood as follows: the more repulsive the potential is at the origin, the higher the dimension of local minimizers will be.

Potentials satisfying (3) have a singular Laplacian at 0 and we refer to them as strongly repulsive at the origin. The second main result is devoted to potentials which are mildly repulsive at the origin, that is potentials whose Laplacian does not blow up at the origin. To be more precise we show that if

$$
W(x) \sim-|x|^{\alpha} \quad \text { as } x \rightarrow 0 \quad \text { for some } \alpha>2
$$

then a local minimizer of the interaction energy cannot be concentrated on smooth manifolds of any dimension except 0 -dimensional sets. The exact hypotheses on $W$, as well as the precise meaning of (4), can be found in Theorem 2. Note that this result suggests that local minimizers of the interaction energy of mildly repulsive potentials have zero Hausdorff dimension - however we are currently unable to prove this stronger result.

Summarizing, in this paper we show that if the Laplacian of the potential behaves like $-1 /|x|^{\beta}$ around the origin, with $0<\beta<N$, then the dimension of minimizers is at least $\beta$ and if the Laplacian does not blow up at the origin, then the dimension is zero, see the precise statement in Theorems 1 and 2. This is illustrated in the case of two dimensions $(N=2)$ in Table 1 , where we show some local minimizers of $E_{W}$ with interaction potentials of the form

$$
W(x)=-\frac{|x|^{\alpha}}{\alpha}+\frac{|x|^{\gamma}}{\gamma} \quad \alpha<\gamma
$$

so that $W(x) \sim-\frac{|x|^{\alpha}}{\alpha}$ and $\Delta W(x) \sim-\frac{1}{|x|^{\beta}}$ with $\beta=2-\alpha$ as $x \rightarrow 0$.

- Subfigure (a): $\alpha=2.5$ and $\gamma=15$. The support of the minimizer has zero Hausdorff dimension in agreement with Theorem 2. Actually, in this particular case it is supported just on 3 points.

- Subfigure (b) and (c): we consider two examples where the potentials have the same behavior at the origin, $\alpha=1.5$, but different attractive long range behavior $(\gamma=7$ and 2 respectively). Theorem 11 shows that the Hausdorff dimension of the support must be greater or equal to $\beta=2-\alpha=0.5$. Indeed, the minimizer for the first example has a one-dimensional support on three curves whereas the minimizer for the second example has two-dimensional support.

- Subfigure (d): $\alpha=0.5$ and $\gamma=5$. Theorem 1 proves that the Hausdorff dimension of the support must be greater than $\beta=2-\alpha=1.5$. The numerical simulation demonstrates that it has dimension two.

In our extensive numerical experiments using gradient descent methods we never observed minimizers with a support that might be of non-integer Hausdorff dimension.

In most of this paper, we will consider local minimizers for the topology induced by the transport distance $d_{\infty}$ (see section 2 for a definition of $d_{\infty}$ ). This topology is indeed the natural one to 


\begin{tabular}{|c|c|c|c|}
\hline & $\operatorname{Dim}=0$ & $\operatorname{Dim}=1$ & $\operatorname{Dim}=2$ \\
\hline$\alpha=2.5$ & (a) & & \\
\hline$\alpha=1.5$ & & (b) & (c) \\
\hline$\alpha=0.5$ & & & (d) \\
\hline
\end{tabular}

TABLE 1. Local minimizers of the interaction energy $E_{W}^{n}$ for various potentials $W(x)$. In these computations $n=10,000$. When $\Delta W$ does not blow-up at the origin (Case a) the Hausdorff dimension of the the support of minimizers is zero. When $\Delta W \sim-1 /|x|^{\beta}$ as $x \rightarrow 0,0<\beta<N$ (Cases b,c,d) the Hausdorff dimension of the the support of minimizers is greater or equal to $\beta$.

consider. In particular, gradient descent numerical methods based on particles typically lead to local minimizers for the $d_{\infty}$-topology. Moreover the topology induced by $d_{\infty}$ is the finest topology among the ones induced by $d_{p}, 1 \leq p \leq \infty$ (see section 2 for a definition of $d_{p}$ ). As a consequence local minimizers in the $d_{p}$-topology are automatically local minimizers in the $d_{\infty}$-topology, and thus they are also covered by our study. In Section 5 we will discuss in more detail these questions.

Let us finally mention that the gradient flow of the energy $E_{W}$ in the Wasserstein sense $d_{2}$ [12, 1, 13] has been extensively studied in recent years [24, 7, 5, 6, 8, 11, 10, 9, 17, 18, 30, 3, 2]. It leads to the nonlocal interaction equation

$$
\frac{\partial \mu}{\partial t}+\operatorname{div}(\mu v)=0 \quad, \quad v=-\nabla W * \mu
$$

where $\mu(t, x)=\mu_{t}(x)$ is the probability or mass density of particles at time $t$ and at location $x \in \mathbb{R}^{N}$, and $v(t, x)$ is the velocity of the particles. Stability properties of steady states for (6) with repulsive-attractive potentials have only been analyzed very recently. In $[3$ we gave conditions for radial stability/instability of particular local minimizers. We should also mention that the one 
dimensional case was analyzed in detail in [17, 18. Well-posedness theories for these repulsiveattractive potentials in various functional settings have been provided in [24, 1, 8, 11, 9, 3]. Stable steady states of (6) under certain set of perturbations are expected to be local minimizers of the energy functional (1) in a topology to be specified. Actually, this topology should determine the set of admissible perturbations. As already mentioned, the $d_{\infty}$-stability is the one typically studied by performing equal mass particles simulations.

Finally, we can now interpret our dimensionality result in terms of the nonlocal evolution equation (6). The heuristic idea behind the implication: (3) with $0<\beta<N$ implies dimensionality larger than $\beta$ of the support of local minimizers of $E_{W}$; can be understood in terms of the divergence of the velocity field in (6). In fact, it is straightforward to check that the divergence of the velocity field generated by a uniform density localized over a smooth manifold of dimension $k$ is $+\infty$ on the manifold if and only if $k<\beta$ (this is equivalent to non-integrability of $-\Delta W$ on manifolds of dimension $k$ ). Heuristically, if $\operatorname{div} v=-\Delta W * \mu$ associated to $\mu$ diverges on its support the density has a strong tendency to spread, the configuration is not stable and then $\mu$ is not a local minimizer. Therefore, we can reinterpret our result in Theorem 1 as follows: local minimizers of (1) have to be supported on manifolds where the divergence of their generated velocity field is not $+\infty$.

The plan of the paper is as follows. Section 2 will be devoted to the necessary background in optimal transport theory and notations. Strongly repulsive potentials are treated in Section 3 while mildly repulsive potentials are analyzed in Section 4. In Section 5, for the smaller subset of local minimizers in the $d_{2}$-topology, we show that we can use an Euler-Lagrange approach in the spirit of [4] to derive some properties of these minimizers. Extensive numerical tests as well as details of the algorithm used in order to minimize $E_{W}^{n}$ are reported in Section 6.

\section{Preliminaries in Transport Distances}

We denote by $\mathcal{B}\left(\mathbb{R}^{N}\right)$ the family of Borel subsets of $\mathbb{R}^{N}$. Given a set $A \in \mathcal{B}\left(\mathbb{R}^{N}\right)$, its Lebesgue measure is denoted by $|A|$. We denote by $\mathcal{M}\left(\mathbb{R}^{N}\right)$ the set of (nonnegative) Borel measures on $\mathbb{R}^{N}$ and by $\mathcal{P}\left(\mathbb{R}^{N}\right)$ the set of Borel probability measures on $\mathbb{R}^{N}$. The support of $\mu \in \mathcal{M}\left(\mathbb{R}^{N}\right)$, denoted by $\operatorname{supp}(\mu)$, is the closed set defined by

$$
\operatorname{supp}(\mu):=\left\{x \in \mathbb{R}^{N}: \mu(B(x, \epsilon))>0 \text { for all } \epsilon>0\right\} .
$$

A measure $\rho \in \mathcal{M}\left(\mathbb{R}^{N}\right)$ is said to be a part of $\mu$ if $\rho(A) \leq \mu(A)$ for all $A \in \mathcal{B}\left(\mathbb{R}^{N}\right)$ and it is not identically zero. This terminology is justified by the fact that if $\rho$ is a part of $\mu$, then $\mu$ can be written $\mu=\rho+\nu$ for some $\nu \in \mathcal{M}\left(\mathbb{R}^{N}\right)(\nu=\mu-\rho$ to be more precise). We will say that a probability measure $\mu \in \mathcal{P}\left(\mathbb{R}^{N}\right)$ can be decomposed as a convex combination of $\mu_{0}, \mu_{1} \in \mathcal{P}\left(\mathbb{R}^{N}\right)$ if there exists $0 \leq m_{0}, m_{1} \leq 1$ with $m_{0}+m_{1}=1$ such that $\mu=m_{0} \mu_{0}+m_{1} \mu_{1}$.

Let us introduce some notation related to the interaction potential energy. We denote by $B_{W}$ : $\mathcal{P}\left(\mathbb{R}^{N}\right) \times \mathcal{P}\left(\mathbb{R}^{N}\right) \rightarrow(0,+\infty]$ the bilinear form defined by

$$
B_{W}\left[\mu_{1}, \mu_{2}\right]:=\frac{1}{2} \iint_{\mathbb{R}^{N} \times \mathbb{R}^{N}} W(x-y) d \mu_{1}(x) d \mu_{2}(y) .
$$

Obviously we have that $E_{W}[\mu]=B_{W}[\mu, \mu]$. Let us define the shortcut notation $T_{W}\left[\mu_{1}, \mu_{2}\right]:=$ $E_{W}\left[\mu_{1}\right]-2 B_{W}\left[\mu_{1}, \mu_{2}\right]+E_{W}\left[\mu_{2}\right]$ which will often occur in several computations. For notational simplicity, we will drop the subscript for $E_{W}, B_{W}$, and $T_{W}$ in detailed proofs while kept in the main statements.

Let us give a brief self-contained summary of the main concepts related to distances between measures in optimal transport theory, we refer to [34, 20, 25] for further details. A probability measure $\pi$ on the product space $\mathbb{R}^{N} \times \mathbb{R}^{N}$ is said to be a transference plan between $\mu \in \mathcal{P}\left(\mathbb{R}^{N}\right)$ and $\nu \in \mathcal{P}\left(\mathbb{R}^{N}\right)$ if

$$
\pi\left(A \times \mathbb{R}^{N}\right)=\mu(A) \quad \underset{4}{\text { and }} \quad \pi\left(\mathbb{R}^{N} \times A\right)=\nu(A)
$$


for all $A \in \mathcal{B}\left(\mathbb{R}^{N}\right)$. If $\mu, \nu \in \mathcal{P}\left(\mathbb{R}^{N}\right)$, then

$$
\Pi(\mu, \nu):=\left\{\pi \in \mathcal{P}\left(\mathbb{R}^{N} \times \mathbb{R}^{N}\right):(8) \text { holds for all } A \in \mathcal{B}\left(\mathbb{R}^{N}\right)\right\}
$$

denotes the set of admissible transference plans between $\mu$ and $\nu$. Informally, if $\pi \in \Pi(\mu, \nu)$ then $d \pi(x, y)$ measures the amount of mass transferred from location $x$ to location $y$. With this interpretation in mind note that $\sup _{(x, y) \in \operatorname{supp}(\pi)}|x-y|$ represents the maximum distance that an infinitesimal element of mass from $\mu$ is moved by the transference plan $\pi$. We will work with the $\infty$-Wasserstein distance $d_{\infty}$ between two probability measures $\mu, \nu$ defined by

$$
d_{\infty}(\mu, \nu)=\inf _{\pi \in \Pi(\mu, \nu)} \sup _{(x, y) \in \operatorname{supp}(\pi)}|x-y|,
$$

which can take infinite values, but it is obviously finite for compactly supported measures. This distance induces a complete metric structure restricted to the set of probability measure with finite moments of all orders, $\mathcal{P}_{\infty}\left(\mathbb{R}^{N}\right)$, as proven in [20].

We remind that for $1 \leq p<\infty$ the distance $d_{p}$ between two measures $\mu$ and $\nu$ is defined by

$$
d_{p}^{p}(\mu, \nu)=\inf _{\pi \in \Pi(\mu, \nu)}\left\{\iint_{\mathbb{R}^{N} \times \mathbb{R}^{N}}|x-y|^{p} d \pi(x, y)\right\} .
$$

Note that $d_{p}(\mu, \nu)<\infty$ for $\mu, \nu \in \mathcal{P}_{p}\left(\mathbb{R}^{N}\right)$ the set of probability measures with finite moments of order $p$. Since $d_{p}(\mu, \nu)$ is increasing as a function of $1 \leq p<\infty$, one can show that it converges to $d_{\infty}(\mu, \nu)$ as $p \rightarrow \infty$. Since the distances are ordered with respect to $p$, it is obvious that the topologies are also ordered. More precisely, open sets for $d_{p}$ are always open sets for $d_{\infty}$, and thus, $d_{\infty}$ induces the finest topology among $d_{p}, 1 \leq p \leq \infty$. More properties of the distance $d_{\infty}$ can be seen in [25].

Given $\mathcal{T}: \mathbb{R}^{N} \longrightarrow \mathbb{R}^{N}$ measurable, we say that $\nu$ is the push-forward of $\mu$ through $\mathcal{T}, \nu=\mathcal{T} \# \mu$, if $\nu[A]:=\mu\left[\mathcal{T}^{-1}(A)\right]$ for all measurable sets $A \subset \mathbb{R}^{N}$, equivalently

$$
\int_{\mathbb{R}^{N}} \varphi(x) d \nu(x)=\int_{\mathbb{R}^{N}} \varphi(\mathcal{T}(x)) d \mu(x)
$$

for all $\varphi \in C_{b}\left(\mathbb{R}^{N}\right)$. In case there is a map $\mathcal{T}: \mathbb{R}^{N} \longrightarrow \mathbb{R}^{N}$ transporting $\mu$ onto $\nu$, i.e. $\mathcal{T} \# \mu=\nu$, we immediately obtain

$$
d_{\infty}(\mu, \nu) \leq \sup _{y \in \operatorname{supp}(\mu)}|y-\mathcal{T}(y)|
$$

This comes from (9), by using the transference plan $\pi_{\mathcal{T}}=\left(\mathbb{1}_{\mathbb{R}^{N}} \times \mathcal{T}\right) \# \mu$.

Lemma 1. Assume that $\mu, \tilde{\mu} \in \mathcal{P}\left(\mathbb{R}^{N}\right)$ are two convex combinations: $\mu=m_{0} \mu_{0}+m_{1} \mu_{1}$ and $\tilde{\mu}=m_{0} \tilde{\mu}_{0}+m_{1} \mu_{1}$, where $\mu_{0}$ and $\tilde{\mu}_{0}$ are supported in $B\left(x_{0}, \epsilon\right)$ for some $x_{0} \in \mathbb{R}^{N}$ and $\epsilon>0$. Then $d_{\infty}(\mu, \tilde{\mu}) \leq 2 \epsilon$.

Proof. Let $\pi_{1} \in \Pi\left(\mu_{1}, \mu_{1}\right)$ be the transport plan induced by the identity map, that is

$$
\iint_{\mathbb{R}^{N} \times \mathbb{R}^{N}} \phi(x, y) d \pi_{1}(x, y)=\int_{\mathbb{R}^{N}} \phi(x, x) d \mu_{1}(x)
$$

and let $\pi_{0} \in \Pi\left(\mu_{0}, \tilde{\mu}_{0}\right)$ be any transport plan between $\mu_{0}$ and $\tilde{\mu}_{0}$. Note that $\pi=m_{0} \pi_{0}+m_{1} \pi_{1} \subset$ $\Pi(\mu, \tilde{\mu})$ and $\operatorname{supp}(\pi)=\operatorname{supp}\left(\pi_{0}\right) \cup \operatorname{supp}\left(\pi_{1}\right)$. Since $\pi_{1}$ is supported on the diagonal we have $\sup _{(x, y) \in \operatorname{supp}\left(\pi_{1}\right)}|x-y|=0$. On the other hand, $\operatorname{supp}\left(\pi_{0}\right) \subset \operatorname{supp}\left(\mu_{0}\right) \times \operatorname{supp}\left(\tilde{\mu}_{0}\right) \subset B\left(x_{0}, \epsilon\right) \times$ $B\left(x_{0}, \epsilon\right)$ and therefore $\sup _{(x, y) \in \operatorname{supp}\left(\pi_{0}\right)}|x-y| \leq 2 \epsilon$. We conclude that $\sup _{(x, y) \in \operatorname{supp}(\pi)}|x-y| \leq 2 \epsilon$ which implies $\inf _{\pi \in \Pi(\nu, \rho)} \sup _{(x, y) \in \operatorname{supp}(\pi)}|x-y| \leq 2 \epsilon$. 


\section{LOWER BOUND ON THE HAUSDORFF DIMENSION OF THE SUPPORT}

In this section we consider potentials which are strongly repulsive at the origin and we prove that if $\Delta W \sim-1 /|x|^{\beta}$ as $x \rightarrow 0,0<\beta<N$, then the Hausdorff dimension of the support of local minimizers of the interaction energy is greater or equal to $\beta$. Actually our result is slightly stronger: we prove that if $\mu$ is a local minimizer then the support of any part of $\mu$ has Hausdorff dimension greater or equal to $\beta$. Let us illustrate the importance of controlling not only the dimension of $\mu$, but also the dimension of the parts of $\mu$. Suppose for example that $\Delta W \sim-1 /|x|$ as $x \rightarrow 0$, then our result implies that any part of $\mu$ has Hausdorff dimension greater or equal to 1 . As a consequence $\mu$ can not have an atomic part. If $\Delta W \sim-1 /|x|^{1.5}$ as $x \rightarrow 0$ then $\mu$ can not have a part concentrated on a curve and so on.

3.1. Hypotheses and statement of the main result. In this section, we will assume that the potential $W: \mathbb{R}^{N} \rightarrow(-\infty,+\infty]$ satisfies the following hypotheses:

(H1) $W$ is bounded from below.

(H2) $W$ is lower semicontinuous (l.s.c.).

(H3) $W$ is uniformly locally integrable: there exists $M>0$ such that $\int_{B(x, 1)} W(y) d y \leq M$ for all $x \in \mathbb{R}^{N}$.

In order to state the main results of this section we will also need the following two definitions:

Definition 1 (Generalized Laplacian). Suppose $W: \mathbb{R}^{N} \rightarrow(-\infty,+\infty]$ is locally integrable. The approximate Laplacian of $W$ is defined by

$$
-\Delta^{\epsilon} W(x):=\frac{2(N+2)}{\epsilon^{2}}\left(W(x)-f_{B(0, \epsilon)} W(x+y) d y\right),
$$

where $f_{B\left(x_{0}, r\right)} f(x) d x$ stands for the average of $f$ over the ball of radius $r$ centered at $x_{0}$, and the generalized Laplacian of $W$ is defined by

$$
-\Delta^{0} W(x):=\liminf _{n \rightarrow \infty}\left\{-\Delta^{(1 / n)} W(x)\right\} .
$$

Definition 2 ( $\beta$-repulsive potential). Suppose $W: \mathbb{R}^{N} \rightarrow(-\infty,+\infty]$ is locally integrable. $W$ is said to be $\beta$-repulsive at the origin if there exists $\epsilon>0$ and $C>0$ such that

$$
\begin{aligned}
& -\Delta^{0} W(x) \geq \frac{C}{|x|^{\beta}} \quad \text { for all } 0<|x|<\epsilon \\
& -\Delta^{0} W(0)=+\infty .
\end{aligned}
$$

By doing a Taylor expansion one can easily check that $\Delta^{0} W(x)=\Delta W(x)$ wherever $W$ is twice differentiable. In particular if $W$ is twice differentiable away from the origin as it is often the case for potentials of interest, then (10) simply means that $-\Delta W(x) \geq C /|x|^{\beta}$ for all $0<|x|<\epsilon$. The terminology " $\beta$-repulsive" is justified by the fact that the rate at which $\Delta^{0} W(x)$ goes to $-\infty$ as $x$ approaches the origin quantifies the repulsive strength of the potential at the origin, therefore the greater $\beta$ is the more repulsive the potential is around the origin. This is the rigorous mathematical formulation of what we meant in (3). Additionally to hypotheses (H1)-(H3), we will need the following technical assumption on the potential $W$ :

(H4) There exists $C^{*}>0$ such that

$$
\Delta^{\epsilon} W(x)<C^{*} \quad \forall x \in \mathbb{R}^{N} \text { and } \forall \epsilon \in(0,1) .
$$

We are now ready to state the main theorems of this section: 
Theorem 1. Suppose $W$ satisfies (H1)-(H4) and let $\mu$ be a compactly supported local minimizer of the interaction energy with respect to the topology induced by $d_{\infty}$. If $W$ is $\beta$-repulsive at the origin, $0<\beta<N$, then the Hausdorff dimension of the support of any part of $\mu$ is greater or equal to $\beta$.

Remark 1. Observe that (H3) and (H4) are conditions which restrict the growth of the potential and its derivatives at $\infty$. For instance, a potential growing algebraically at $\infty$ does not satisfy those assumptions. However, if we are only interested in the dimensionality of the support for compactly supported local minimizers, Theorem 1 holds under weaker assumptions not restricting the growth of the potential at $\infty$. Namely, (H3) and (H4) can be substituted by (H3-loc) and (H4-loc):

(H3-loc) $W$ is locally integrable.

(H4-loc) For every compact subset $K$ of $\mathbb{R}^{N}$ there exists $C_{K}^{*}>0$ such that

$$
\Delta^{\epsilon} W(x)<C_{K}^{*} \quad \forall x \in K \text { and } \forall \epsilon \in(0,1),
$$

with obvious changes in the proof.

Remark 2. In Theorem 1 (resp. Remark 1) potential $W$ is assumed to be $\beta$-repulsive at the origin and to satisfy hypotheses $(\mathrm{H} 1)-(\mathrm{H} 4)$ (resp. (H1)-(H2)-(H3-loc)-(H4-loc)). Whereas hypotheses (H1)-(H3) (resp. (H1)-(H2)-(H3-loc)) are easily verified for a given potential, hypotheses $(\mathrm{H} 4)$ or (H4-loc) and the $\beta$-repulsivity are not as transparent. To clarify the meaning of these more technical assumptions let us consider the case where $W$ is smooth away from the origin and satisfies

$$
-\Delta W(x) \geq \frac{C}{|x|^{\beta}} \quad \text { for all } 0<|x|<\epsilon
$$

for some $0<\beta<N$. Such a potential satisfies (10) as pointed out in the comment after Definition 2. Moreover most potentials of interest satisfying (12) will also satisfy (11) and either (H4) or (H4-loc), but of course this need to be checked case by case. In subsection 3.3 we consider some typical repulsive-attractive potentials satisfying (12) and we show that they satisfy (11) and either (H4) or (H4-loc) depending on their behavior at infinity.

3.2. Proof of Theorem 1. First note that without loss of generality we can replace hypothesis $(H 1)$ by

(H1') $W$ is nonnegative

since adding a constant to the potential $W$ does not affect the local minimizers of $E_{W}$. The following lemma is classical:

Lemma 2. Suppose $W$ satisfies (H1') and (H2) and let $\mu \in \mathcal{P}\left(\mathbb{R}^{N}\right)$. Then the function $V_{\mu}: \mathbb{R}^{N} \rightarrow$ $[0,+\infty]$ defined by

is lower semicontinuous.

$$
V_{\mu}(x)=(W * \mu)(x)=\int_{\mathbb{R}^{N}} W(x-y) d \mu(y)
$$

Proof. Suppose $x_{n} \rightarrow x$, then by Fatou's lemma we have

$$
\begin{aligned}
V_{\mu}(x)=\int_{\mathbb{R}^{N}} W(x-y) d \mu(y) & \leq \int_{\mathbb{R}^{N}} \liminf _{n} W\left(x_{n}-y\right) d \mu(y) \\
& \leq \liminf _{n} \int_{\mathbb{R}^{N}} W\left(x_{n}-y\right) d \mu(y)=\liminf _{n} V_{\mu}\left(x_{n}\right)
\end{aligned}
$$

as desired.

Suppose now that $W$ satisfies (H1')-(H4). Note that hypothesis (H4) implies that $-\Delta^{0} W \geq-C^{*}$ and as a consequence, for any $\mu \in \mathcal{P}\left(\mathbb{R}^{N}\right)$, the function

$$
\left(-\Delta^{0} W * \mu\right)(x)=\int_{\mathbb{R}^{N}}\left(-\Delta^{0} W\right)(x-y) d \mu(y)=\int_{\mathbb{R}^{N}}\left[\left(-\Delta^{0} W\right)(x-y)+C^{*}\right] d \mu(y)-C^{*}
$$


is defined for all $x$ and $-\Delta^{0} W * \mu: \mathbb{R}^{N} \rightarrow\left[-C^{*},+\infty\right]$.

Lemma 3. Suppose that $W$ satisfies (H1')-(H4) and let $\mu \in \mathcal{P}\left(\mathbb{R}^{N}\right)$. If $x_{0}$ is a local min of $V_{\mu}=W * \mu$, in the sense that there exists $\epsilon_{0}>0$ such that

$$
V_{\mu}\left(x_{0}\right) \leq V_{\mu}(x) \text { for almost every } x \in B\left(x_{0}, \epsilon_{0}\right),
$$

then $\left(\Delta^{0} W * \mu\right)\left(x_{0}\right) \geq 0$.

Proof. Assume that $x_{0}$ satisfies 13). We first show that $V_{\mu}\left(x_{0}\right)<+\infty$. If it were not the case we would have that $V_{\mu}=+\infty$ a.e. in $B\left(x_{0}, \epsilon_{0}\right)$. But hypothesis (H3) and Fubini's Theorem imply that

$$
\int_{B\left(x_{0}, 1\right)} V_{\mu}(x) d x \leq \int_{\mathbb{R}^{N}} \int_{B\left(x_{0}-y, 1\right)} W(z) d z d \mu(y) \leq M
$$

and therefore $V_{\mu}$ is finite almost everywhere in $B\left(x_{0}, 1\right)$, contradicting the fact that $V_{\mu}=+\infty$ a.e. in $B\left(x_{0}, \epsilon_{0}\right)$. Now, for $\epsilon \leq \epsilon_{0}$ we have

$$
\begin{aligned}
0 & \leq \frac{2(N+2)}{\epsilon^{2}}\left(f_{B(0, \epsilon)} V_{\mu}\left(x_{0}+x\right) d x-V_{\mu}\left(x_{0}\right)\right) \\
& =\frac{2(N+2)}{\epsilon^{2}}\left(\int_{\mathbb{R}^{N}} f_{B(0, \epsilon)} W\left(x_{0}+x-y\right) d x d \mu(y)-\int_{\mathbb{R}^{N}} W\left(x_{0}-y\right) d \mu(y)\right) .
\end{aligned}
$$

Note that hypothesis (H4) implies that

$$
f_{B(0, \epsilon)} W\left(x_{0}+x-y\right) d x \leq W\left(x_{0}-y\right)+\frac{C^{*} \epsilon^{2}}{2(d+2)} .
$$

Since $V_{\mu}\left(x_{0}\right)<+\infty$, the functions $y \mapsto W\left(x_{0}-y\right)$ and $y \mapsto f_{B(0, \epsilon)} W\left(x_{0}+x-y\right) d x$ are $\mu$-integrable and the difference of the integrals in (14) is equal to the integral of the difference. Therefore we have:

$$
0 \leq \int_{\mathbb{R}^{N}} \frac{2(d+2)}{\epsilon^{2}}\left(f_{B(0, \epsilon)} W\left(x_{0}-y+x\right) d x-W\left(x_{0}-y\right)\right) d \mu(y)=\int_{\mathbb{R}^{N}} \Delta^{\epsilon} W\left(x_{0}-y\right) d \mu(y) .
$$

Because of hypothesis (H4), we have that $-\Delta^{\epsilon} W+C^{*} \geq 0$ for all $\epsilon \in(0,1)$. Therefore using Fatou's Lemma and (15):

$$
\begin{aligned}
\int_{\mathbb{R}^{N}} \liminf _{n \rightarrow \infty} & \left\{-\Delta^{(1 / n)} W\left(x_{0}-y\right)+C^{*}\right\} d \mu(y) \\
& \leq \liminf _{n \rightarrow \infty} \int_{\mathbb{R}^{N}}\left[-\Delta^{(1 / n)} W\left(x_{0}-y\right)+C^{*}\right] d \mu(y) \leq C^{*},
\end{aligned}
$$

that is, $\left(\Delta^{0} W * \mu\right)\left(x_{0}\right) \geq 0$.

Proposition 1. Suppose that $W$ satisfies (H1')-(H2)-(H3). Let $\mu$ be a local minimizer of the interaction energy with respect to the $d_{\infty}$ and assume that $E[\mu]<+\infty$. Then any point $x_{0} \in$ $\operatorname{supp}(\mu)$ is a local minimizer of $V_{\mu}$, in the sense that there exists $\epsilon_{0}>0$ such that

$$
V_{\mu}\left(x_{0}\right) \leq V_{\mu}(x) \text { for almost every } x \in B\left(x_{0}, \epsilon_{0}\right) .
$$

Proof. We argue by contradiction. Assume that there exists $x_{0} \in \operatorname{supp}(\mu)$ which is not a local minimum of $V_{\mu}$. Fix $\epsilon>0$. Then there exists a set $A \subset B\left(x_{0}, \epsilon\right)$ of positive Lebesgue measure, such that for $x \in A, V_{\mu}(x)<V_{\mu}\left(x_{0}\right)$. The set $A$ can be written as follows:

$$
A=\cup_{n=1}^{\infty}\left\{x \in A ; V_{\mu}(x) \leq V_{\mu}\left(x_{0}\right)-1 / n\right\},
$$


that is $A$ is an increasing union of measurable sets. Thanks to the continuity from below of the Lebesgue measure, it implies that

$$
0<|A|=\lim _{n \rightarrow \infty}\left|\left\{x \in A ; V_{\mu}(x) \leq V_{\mu}\left(x_{0}\right)-1 / n\right\}\right|,
$$

and there exists $n_{0}$ such that $\tilde{A}:=\left\{x \in A ; V_{\mu}(x) \leq V_{\mu}\left(x_{0}\right)-1 / n_{0}\right\}$ is of positive Lebesgue measure. Thanks to the lower semicontinuity of $V_{\mu}$, there exists $\eta \in(0, \epsilon)$ such that

$$
\inf _{B\left(x_{0}, \eta\right)} V_{\mu} \geq V_{\mu}\left(x_{0}\right)-\frac{1}{2 n_{0}} \geq \sup _{\tilde{A}} V_{\mu}+\frac{1}{2 n_{0}} .
$$

Notice that $x_{0} \in \operatorname{supp}(\mu)$ implies $\mu\left(B\left(x_{0}, \eta\right)\right)>0$. We can therefore define the probability measures $\mu_{0}, \mu_{\tilde{A}}$ by

$$
\mu_{0}(B)=\frac{1}{m_{0}} \mu\left(B \cap B\left(x_{0}, \eta\right)\right), \quad \mu_{\tilde{A}}(B)=\frac{1}{|\tilde{A}|}|B \cap \tilde{A}|
$$

for any Borel set $B \in \mathcal{B}\left(\mathbb{R}^{N}\right)$, where $m_{0}:=\mu\left(B\left(x_{0}, \eta\right)\right)$. Let us now write $\mu$ as a convex combination $\mu=m_{0} \mu_{0}+m_{1} \mu_{1}$, and define the curve of measures

$$
\mu_{t}=\left(m_{0}-t\right) \mu_{0}+t \mu_{\tilde{A}}+m_{1} \mu_{1} .
$$

It is clear by construction that $\mu_{t} \in \mathcal{P}\left(\mathbb{R}^{N}\right)$ for $t \in\left[0, m_{0}\right]$. Note that $\mu_{t}$ is obtained from $\mu$ by transporting an amount $t$ of mass from the region $B\left(x_{0}, \eta\right)$ and by distributing it uniformly in the region $\tilde{A}$. Since both $B\left(x_{0}, \eta\right)$ and $\tilde{A}$ are contained in $B\left(x_{0}, \epsilon\right)$, the mass is transported by a distance smaller than $2 \epsilon$ and therefore we have $d_{\infty}\left(\mu, \mu_{t}\right) \leq 2 \epsilon$, see Lemma 1 for details. Inequality (16) shows that the function $V_{\mu}$ is greater on the region $B\left(x_{0}, \eta\right)$ than on the region $\tilde{A}$, therefore one would expect that transporting mass from one region to the other will decrease the interaction energy. Indeed we will show that $E\left[\mu_{t}\right]<E[\mu]$ for $t$ small enough. Since $\epsilon$ was arbitrary, this will imply that we can always find a probability measure arbitrarily close to $\mu$ (in the sense of the $d_{\infty}$ ) with strictly smaller energy. This is a contradiction concluding the proof.

We are left to show that $E\left[\mu_{t}\right]<E[\mu]$ for $t$ small enough. Since $0 \leq E[\mu]<\infty$ and given by

$$
E[\mu]=m_{0}^{2} E\left[\mu_{0}\right]+2 m_{0} m_{1} B\left[\mu_{0}, \mu_{1}\right]+m_{1}^{2} E\left[\mu_{1}\right]
$$

then the three terms $E\left[\mu_{0}\right], B\left[\mu_{0}, \mu_{1}\right]$ and $E\left[\mu_{1}\right]$ are all positive and finite. Note that $E\left[\mu_{\tilde{A}}\right]$ is also finite: indeed, since $W$ is locally integrable by (H3), we have

$$
E\left[\mu_{\tilde{A}}\right]=\iint_{\mathbb{R}^{N} \times \mathbb{R}^{N}} W(x-y) d \mu_{\tilde{A}}(x) d \mu_{\tilde{A}}(y) \leq \frac{1}{|\tilde{A}|^{2}} \iint_{B\left(x_{0}, \epsilon\right) \times B\left(x_{0}, \epsilon\right)} W(x-y) d x d y<+\infty .
$$

From (16) and the fact that $B\left[\mu, \mu_{0}\right] \leq \frac{1}{m_{0}} E[\mu]<+\infty$, we also have that

$$
B\left[\mu, \mu_{\tilde{A}}\right]+\frac{1}{2 n_{0}} \leq B\left[\mu, \mu_{0}\right]<+\infty .
$$

Using all these, we can show that all combinations of the bilinear form $B[\cdot, \cdot]$ for the measures $\mu_{0}$, $\mu_{1}$, and $\mu_{\tilde{A}}$ are finite:

$$
\begin{array}{ll}
E\left[\mu_{0}\right]<+\infty \quad, \quad E\left[\mu_{1}\right]<+\infty \quad, \quad E\left[\mu_{\tilde{A}}\right]<+\infty, \quad B\left[\mu_{1}, \mu_{0}\right]<+\infty, \\
B\left[\mu_{\tilde{A}}, \mu_{0}\right] \leq \frac{1}{m_{0}} B\left[\mu_{\tilde{A}}, \mu\right]<+\infty \quad, \quad B\left[\mu_{\tilde{A}}, \mu_{1}\right] \leq \frac{1}{m_{1}} B\left[\mu_{\tilde{A}}, \mu\right]<+\infty,
\end{array}
$$

where we have used (17) in order to obtain (19). Note that in 19$)$ we have assumed $m_{1} \neq 0$. If $m_{1}=0$ then $\mu_{1}$ can be chosen to be zero and therefore $B\left[\mu_{\tilde{A}}, \mu_{1}\right]<+\infty$ trivially holds. Using 
(18)-(19), we are allowed to expand $E\left[\mu_{t}\right]$ as:

$$
\begin{aligned}
E\left[\mu_{t}\right]= & E\left[\left(m_{0}-t\right) \mu_{0}+m_{1} \mu_{1}+t \mu_{\tilde{A}}\right] \\
= & \left(m_{0}-t\right)^{2} E\left[\mu_{0}\right]+m_{1}^{2} E\left[\mu_{1}\right]+t^{2} E\left[\mu_{\tilde{A}}\right] \\
& +2\left(m_{0}-t\right) m_{1} B\left[\mu_{0}, \mu_{1}\right]+2\left(m_{0}-t\right) t B\left[\mu_{0}, \mu_{\tilde{A}}\right]+2 m_{1} t B\left[\mu_{1}, \mu_{\tilde{A}}\right] \\
= & m_{0}^{2} E\left[\mu_{0}\right]+2 m_{0} m_{1} B\left[\mu_{0}, \mu_{1}\right]+m_{1}^{2} E\left[\mu_{1}\right] \\
& +2 t\left(m_{0} B\left[m_{0}, \mu_{\tilde{A}}\right]+m_{1} B\left[\mu_{1}, \mu_{\tilde{A}}\right]\right)-2 t\left(m_{0} B\left[\mu_{0}, \mu_{0}\right]+m_{1} B\left[\mu_{0}, \mu_{1}\right]\right) \\
& +t^{2} E\left[\mu_{0}\right]+t^{2} E\left[\mu_{\tilde{A}}\right]-2 t^{2} B\left[\mu_{0}, \mu_{\tilde{A}}\right] \\
= & E[\mu]+2 t\left(B\left[\mu_{\tilde{A}}, \mu\right]-B\left[\mu_{0}, \mu\right]\right)+t^{2} T\left[\mu_{0}, \mu_{\tilde{A}}\right] .
\end{aligned}
$$

Note that in the above computations we have only used the bilinearity of $B[\cdot, \cdot]$ on the space of positive measures. However, a formal computation using the bilinearity of $B[\cdot, \cdot]$ on the space of signed measures leads to the same result in a much simpler way:

$$
E\left[\mu_{t}\right]=E\left[\mu-t \mu_{0}+t \mu_{\tilde{A}}\right]=E[\mu]+2 t\left(B\left[\mu_{\tilde{A}}, \mu\right]-B\left[\mu_{0}, \mu\right]\right)+t^{2} T\left[\mu_{0}, \mu_{\tilde{A}}\right] .
$$

To conclude the proof note that because of (17) the term $B\left[\mu_{\tilde{A}}, \mu\right]-B\left[\mu_{0}, \mu\right]$ appearing in (20) is strictly negative and since the term $T\left[\mu_{0}, \mu_{\tilde{A}}\right]=E\left[\mu_{0}\right]-2 B\left[\mu_{0}, \mu_{\tilde{A}}\right]+E\left[\mu_{\tilde{A}}\right]$ is finite we can choose $t$ small enough so that $E\left[\mu_{t}\right]<E[\mu]$. This concludes the proof.

Under the additional assumption that $W$ is not singular at the origin, we can obtain a slightly stronger version of Proposition 1 which will be needed in section 4.

Proposition 2. Assume that $W$ and $\mu$ satisfy the same hypotheses than in Proposition 1. Assume moreover that $W(0)<+\infty$. Then any point $x_{0} \in \operatorname{supp}(\mu)$ is a local minimizer of $V_{\mu}$ in the classical sense and $V_{\mu}$ is constant on any connected compact set $K \subset \operatorname{supp}(\mu)$.

Proof. The proof of the first statement is similar to the proof of Proposition 1. We argue by contradiction: assume that $\mu \in \mathcal{P}\left(\mathbb{R}^{N}\right)$ is a local minimizer of $E[\cdot]$ and that there exists $x_{0} \in \operatorname{supp}(\mu)$ which is not a (classical) local minimum of $V_{\mu}$. Fix $\epsilon>0$, then there exists $x_{a} \in B\left(x_{0}, \epsilon\right)$ such that $V_{\mu}\left(x_{a}\right)<V_{\mu}\left(x_{0}\right)$. But since $V_{\mu}$ is l.s.c. there exists $0<\eta<\epsilon$ such that

$$
V_{\mu}\left(x_{a}\right)<V_{\mu}\left(x_{0}\right) \leq V_{\mu}(x) \text { for all } x \in B\left(x_{0}, \eta\right) .
$$

We then define $\mu_{0}$ and $\mu_{1}$ as in the proof of Proposition 1. The different idea now is to send mass from $\mu_{0}$ to a Dirac Delta at the location $x_{a}$ instead of distributing it evenly over a set $\tilde{A}$ of nonzero Lebesgue measure: instead of letting $\mu_{t}=\left(m_{0}-t\right) \mu_{0}+t \mu_{\tilde{A}}+m_{1} \mu_{1}$ as before, we now define $\mu_{t}=\left(m_{0}-t\right) \mu_{0}+t \delta_{x_{a}}+m_{1} \mu_{1}$. The same expansion leads to

$$
E\left[\mu_{t}\right]=E\left[\mu-t \mu_{0}+t \delta_{x_{a}}\right]=E[\mu]+2 t\left(B\left[\delta_{x_{a}}, \mu\right]-B\left[\mu_{0}, \mu\right]\right)+t^{2} T\left[\mu_{0}, \delta_{x_{a}}\right] .
$$

From (21) we obtain that the term $B\left[\delta_{x_{a}}, \mu\right]-B\left[\mu_{0}, \mu\right]$ is strictly negative. In order to conclude the argument we need the term $T\left[\mu_{0}, \delta_{x_{a}}\right]=E\left[\mu_{0}\right]-2 B\left[\mu_{0}, \delta_{x_{a}}\right]+E\left[\delta_{x_{a}}\right]$ to be finite. Note that $E\left[\delta_{x_{a}}\right]=W(0) / 2$ therefore it is necessary for $W(0)$ to be finite in order to conclude the proof.

We now prove the second statement. We follow classical arguments from potential theory, see [28, Proposition 0.4] for instance. Let $K$ be a connected compact set contained in $\operatorname{supp}(\mu)$ and consider the sets $A=\left\{x \in K: V_{\mu}(x)>\inf _{K} V_{\mu}\right\}$ and $B=\left\{x \in K: V_{\mu}(x)=\inf _{K} V_{\mu}\right\}$. Since $V_{\mu}$ is l.s.c. the set $A$ is open relative to $K$. Let us show that $B$ is also open relative to $K$. We argue by contradiction. Suppose there exists $x_{a} \in B$ such that for every $\epsilon>0$ there exists $x_{0, \epsilon} \in K$ with $\left|x_{a}-x_{0, \epsilon}\right|<\epsilon$ and $V_{\mu}\left(x_{a}\right)<V_{\mu}\left(x_{0, \epsilon}\right)$. Then following the exact same steps as in the proof of the first statement we can construct a probability measure with lower energy than $\mu$ and whose $d_{\infty}$ 
distance to $\mu$ is smaller than $\epsilon$, therefore leading to a contradiction and proving that $B$ is open relative to $K$. Since $K$ is connected then either $A$ or $B$ must be empty. But since $V_{\mu}$ is l.s.c it has to reach its minimum on compact sets and therefore $A=\emptyset$ and $B=K$.

Remark 3. Since $\operatorname{supp}(\mu)$ is closed, the connected component of $\operatorname{supp}(\mu)$ are also closed. So the second statement of Proposition 2 implies that $V_{\mu}$ is constant on any bounded connected component of $\operatorname{supp}(\mu)$. In particular if $\mu$ is compactly supported then $V_{\mu}$ is constant on any connected component of $\operatorname{supp}(\mu)$.

Combining Lemma 3 and Proposition 1 we obtain:

Corolary 1. Suppose that $W$ satisfies (H1')-(H4). If $\mu$ is local minimizer of the interaction energy with respect to $d_{\infty}$ and $E[\mu]<+\infty$, then $\left(\Delta^{0} W * \mu\right)(x) \geq 0$ for all $x \in \operatorname{supp}(\mu)$.

We recall the following result from [16, Theorem 4.13].

Proposition 3. Let $A$ be a Borel subset of $\mathbb{R}^{N}$, and $s \geq 0$. If there exists a probability measure $\mu \in \mathcal{P}\left(\mathbb{R}^{N}\right)$ supported on $A$ such that

$$
\iint_{\mathbb{R}^{N} \times \mathbb{R}^{N}} \frac{d \mu(x) d \mu(y)}{|x-y|^{s}}<\infty
$$

then $\operatorname{dim}_{H} A \geq s$, with $\operatorname{dim}_{H}$ being the Hausdorff dimension of $A$.

We are now ready to prove the main theorem.

Proof of Theorem 1. Let $\rho$ be a nonzero part of $\mu$, that is $\mu=\rho+\nu$ for some nonnegative measure $\nu$. Let $A=\operatorname{supp}(\rho)$ and let us show that $\operatorname{dim}_{H} A \geq \beta$. Choose $\epsilon$ small enough so that 10 holds, choose $x_{0} \in A$ and define the measure

$$
\mu_{0}(B)=\rho\left(B \cap B\left(x_{0}, \epsilon / 2\right)\right) .
$$

Clearly $\mu$ can be written $\mu=\mu_{0}+\mu_{1}$, where $\mu_{0}$ and $\mu_{1}$ are two (nonnegative) measures of mass $m_{0}>0$ and $m_{1} \geq 0$ and where $\mu_{0}$ is supported in $A \cap B\left(x_{0}, \epsilon / 2\right)$. Then from (10) we get:

$$
\begin{aligned}
C \iint_{\mathbb{R}^{N} \times \mathbb{R}^{N}} \frac{d \mu_{0}(x) d \mu_{0}(y)}{|x-y|^{\beta}} \leq \iint_{\mathbb{R}^{N} \times \mathbb{R}^{N}}-\Delta^{0} W(x-y) d \mu_{0}(x) d \mu_{0}(y) \\
\quad=\iint_{\mathbb{R}^{N} \times \mathbb{R}^{N}}-\Delta^{0} W(x-y) d \mu(x) d \mu_{0}(y)-\iint_{\mathbb{R}^{N} \times \mathbb{R}^{N}}-\Delta^{0} W(x-y) d \mu_{1}(x) d \mu_{0}(y) \\
\quad=-\int_{\mathbb{R}^{N} \times \mathbb{R}^{N}}\left(\Delta^{0} W * \mu\right)(y) d \mu_{0}(y)+\iint_{\mathbb{R}^{N} \times \mathbb{R}^{N}} \Delta^{0} W(x-y) d \mu_{1}(x) d \mu_{0}(y) \\
\leq \iint_{\mathbb{R}^{N} \times \mathbb{R}^{N}} \Delta^{0} W(x-y) d \mu_{1}(x) d \mu_{0}(y) \leq C^{*} m_{1} m_{0}<+\infty .
\end{aligned}
$$

We have used the fact that $\Delta^{0} W * \mu$ is nonnegative on the support of $\mu$ from Corollary 1 and that $\Delta^{0} W(x)<C^{*}$ by hypothesis (H4). We then apply Proposition 3 to the probability measure $\mu_{0} / m_{0}$, which is supported on $A$, to obtain $\operatorname{dim}_{H} A \geq \beta$.

3.3. Example of potentials satisfying the hypotheses of Theorem 1. In this subsection we consider the class of potentials

$$
W_{\alpha}(x)=c h_{\alpha}(x)+\psi(x)
$$

where $\psi \in C^{3}\left(\mathbb{R}^{N}\right)$ bounded from below, $c>0$ and $h_{\alpha}: \mathbb{R}^{N} \rightarrow(-\infty, \infty]$ is the power-law function:

$$
h_{\alpha}(x)=-|x|^{\alpha} / \alpha
$$

for $x \neq 0$ and $\alpha \in \mathbb{R}$ with the convention $h_{0}(x)=-\log |x|$. We define $h_{\alpha}(0)=0$ if $\alpha>0$ or $h_{\alpha}(0)=+\infty$ if $\alpha \leq 0$. The potentials $W_{\alpha}$ are typical examples of repulsive-attractive potentials 
behaving like $-|x|^{\alpha} / \alpha$ around the origin. It is trivial to check that $W_{\alpha}$ satisfies (H1)-(H2)-(H3-loc) for any $\alpha>-N$ (in the case $\alpha \geq 0$ the function $\psi$ need to grow fast enough at infinity for hypothesis (H1) to hold). Note also that for $x \neq 0$ we have

$$
-\Delta W_{\alpha}(x)=c \frac{(\alpha+N-2)}{|x|^{2-\alpha}}-\Delta \psi(x)
$$

and therefore if $\alpha+N-2>0$ then $W_{\alpha}$ satisfies 10 from the definition of $\beta$-repulsivity with $\beta=2-\alpha$. The goal of this subsection is to show that $W_{\alpha}$ also satisfies (11) and (H4-loc).

We start by checking (11). An explicit computation gives

$$
-\Delta^{\epsilon} h_{\alpha}(0)=\frac{2(N+2)}{\epsilon^{2}}\left(h_{\alpha}(0)-f_{B(0, \epsilon)} h_{\alpha}(y) d y\right)= \begin{cases}2(N+2) \frac{N}{N+\alpha} \frac{\epsilon^{\alpha-2}}{\alpha} & \text { if } \alpha>0 \\ +\infty & \text { if } 2-N \leq \alpha \leq 0\end{cases}
$$

where we have used the fact that $h_{\alpha}(0)=0$ for $\alpha>0$ and $h_{\alpha}(0)=+\infty$ for $\alpha \leq 0$. Letting $\epsilon \rightarrow 0$ and using the fact that $\Delta \psi(0)$ is finite we obtain

$$
-\Delta^{0} W(0)=+\infty \quad \text { for all } \alpha<2 .
$$

Combining (22) and (23) we see that for $2-N<\alpha<2$ the potential $W_{\alpha}$ is $\beta$-repulsive with $\beta=2-\alpha \in(0, N)$.

We now show that for $\alpha>2-N$ the potentials $W_{\alpha}$ satisfies hypothesis (H4-loc). The key point is that the functions $h_{\alpha}$ are superharmonic for $\alpha>2-N$. Let us recall the definition of superharmonicity:

Definition 3. A lower semicontinuous function $h: \mathbb{R}^{N} \rightarrow(-\infty, \infty]$ is said to be superharmonic on the connected open set $\Omega$ if it is not identically equal to $+\infty$ on $\Omega$ and if

$$
h(x) \geq f_{B(x, r)} h(y) d y
$$

for all $x \in \Omega$ and $r>0$ such that $B(x, r) \subset \Omega$.

We also recall that if $h \in C^{2}(\Omega)$, then $h$ is superharmonic on $\Omega$ if and only if $\Delta h(x) \leq 0$ for all $x \in \Omega$. To see that the functions $h_{\alpha}$ are superharmonic for $\alpha>2-N$, first note that for $x \neq 0$

$$
\Delta h_{\alpha}(x)=-\frac{(\alpha+N-2)}{|x|^{2-\alpha}} \leq 0
$$

Therefore $h_{\alpha}$ is superharmonic on $\mathbb{R}^{N} \backslash\{0\}$ and it can be easily checked that it satisfies the supermean value property at the origin [28, Definition 2.1]. Both together imply that it is actually superharmonic on the full space $\mathbb{R}^{N},[28$, Corollary 2.1]. As a consequence we directly obtain from the definition of the approximate Laplacian that $\Delta^{\epsilon} h_{\alpha}(x) \leq 0$ and therefore

$$
\Delta^{\epsilon} W_{\alpha}=c \Delta^{\epsilon} h_{\alpha}+\Delta^{\epsilon} \psi \leq \Delta^{\epsilon} \psi
$$

To conclude we note that since $\psi \in C^{3}\left(\mathbb{R}^{N}\right)$ a simple Taylor expansion shows that $\Delta^{\epsilon} \psi$ converges uniformly to $\Delta \psi$ on compact sets. Indeed, the expansion gives

$$
\begin{aligned}
\Delta^{\epsilon} \psi(x) & =\frac{2(N+2)}{\epsilon^{2}} f_{B(0, \epsilon)} \psi(x+y)-\psi(x) d y \\
& =\frac{2(N+2)}{\epsilon^{2}} f_{B(0, \epsilon)} y^{T} \nabla \psi(x)+y^{T} H \psi(x) y+O\left(\epsilon^{3}\right) d y \\
& =\frac{2(N+2)}{\epsilon^{2}}\left(\Delta \psi(x) f_{B(0, \epsilon)} y_{1}^{2} d y+O\left(\epsilon^{3}\right)\right) \\
& =\Delta \psi(x)+O(\epsilon)
\end{aligned}
$$


To go from (24) to (25) we have used the fact that most of the terms in the Taylor expansion are equal to zero after integrating on spheres due to symmetry. The only remaining terms are the ones involved in the Laplacian. Note that since the partial derivative of order 3 of $\psi$ are bounded on compact subsets of $\mathbb{R}^{N}$, then the error term is uniform for $x$ in compact sets. Since $\Delta \psi$ is bounded on compact sets, we conclude that for $\alpha>2-N$ the potential $W_{\alpha}$ satisfies (H4-loc).

Moreover, let us point out that if $\psi$ is well behaved at infinity in terms of regularity and growth, then $W_{\alpha}$ satisfies either (H1)-(H4) or (H1)-(H2)-(H3-loc)-(H4-loc). We summarize this discussion in the following proposition:

Proposition 4. If $2-N<\alpha<2$ and if $\psi \in C^{3}\left(\mathbb{R}^{N}\right)$ then $W_{\alpha}$ is $(2-\alpha)$-repulsive around the origin and satisfies (H4-loc).

\section{Mild Repulsion implies 0-Dimensionality}

In this section, we will show that if the potential is mildly repulsive, meaning that it behaves locally near zero like $-|x|^{\alpha}$ with $\alpha>2$, then the support of the measure cannot contain measures concentrated on smooth manifolds of any dimension except 0-dimensional sets.

Definition 4. Let $1 \leq k \leq N$. A probability measure $\mu \in \mathcal{P}\left(\mathbb{R}^{N}\right)$ is said to have a regular $k$-dimensional part if it can be written

$$
\mu=\mu_{1}+\mu_{2}
$$

where $\mu_{1}$ is a nonnegative measure on $\mathbb{R}^{N}$ and $\mu_{2}$ is defined by

$$
\int_{\mathbb{R}^{N}} \psi(x) d \mu_{2}(x)=\int_{\mathcal{M}} \psi(x) f(x) d \sigma(x) \quad \forall \psi \in C_{0}\left(\mathbb{R}^{N}\right)
$$

for some $C^{2}$ manifold $\mathcal{M}$ of dimension $k$ and a non identically zero nonnegative function $f: \mathcal{M} \rightarrow$ $(0,+\infty]$ integrable with respect to the surface measure $d \sigma(x)$ on $\mathcal{M}$. Moreover to avoid pathological situations, we assume that there exists $x_{0} \in \mathcal{M}, c, \kappa>0$ such that

$$
f(x) \geq c \quad \forall x \in \mathcal{M} \cap B\left(x_{0}, \kappa\right) .
$$

We now state the main result of this section:

Theorem 2. Let $W \in C^{2}\left(\mathbb{R}^{N}\right)$ be a radially symmetric potential which is equal to $-|x|^{\alpha} / \alpha$ in a neighborhood of the origin. If $\alpha>2$ then a local minimizer of the interaction energy with respect to $d_{\infty}$ cannot have a $k$-dimensional component for any $1 \leq k \leq N$.

For the above theorem to be true it is not necessary for the potential to be exactly equal to a power law $-|x|^{\alpha}, \alpha>2$, around the origin. It is enough for the potential to behaves like $-|x|^{\alpha}$, $\alpha>2$, at the origin in a precise convexity sense - see Theorem 3 .

4.1. Preliminaries on convexity. To prove Theorem 2, we need some convex analysis concepts, see [13, 1] and the references therein. The term modulus of convexity refers to any convex function $\phi$ on the positive reals satisfying

$$
\begin{array}{ll}
\left(\phi_{0}\right) & \phi:[0, \infty) \longrightarrow \mathbb{R} \text { is continuous and vanishes only at } \phi(0)=0 . \\
\left(\phi_{1}\right) & \phi(x) \geq-k x \text { for some } k<\infty .
\end{array}
$$

Now, we can quantify the convexity of certain functions in terms of a modulus of convexity.

Definition 5. A function $h:[0,+\infty) \rightarrow \mathbb{R}$ is $\phi$-uniformly convex on $(a, b)$ if there exists a modulus of convexity $\phi$ such that

$$
h\left(\frac{r_{1}+r_{2}}{2}\right) \leq \frac{1}{2}\left(h\left(r_{1}\right)+h\left(r_{2}\right)\right)-\frac{1}{4} \int_{0}^{\left|r_{1}-r_{2}\right|} \phi(t) d t,
$$

for all $r_{1}, r_{2} \in(a, b)$. 
A function $h:[0,+\infty) \rightarrow \mathbb{R}$ is $\lambda$-convex on $(a, b)$ if it is $\phi$-uniformly convex with $\phi(s)=\lambda$ s and $\lambda \in \mathbb{R}$.

Note that if $h$ is $\lambda$-convex, then (27) reads

$$
h\left(\frac{r_{1}+r_{2}}{2}\right) \leq \frac{1}{2} h\left(r_{1}\right)+\frac{1}{2} h\left(r_{2}\right)-\frac{\lambda}{8}\left(r_{1}-r_{2}\right)^{2},
$$

for all $r_{1}, r_{2} \in(a, b)$. It is equivalent to assume that the function $h(r)-\frac{\lambda}{2} r^{2}$ is convex on $(a, b)$. The following proposition can be easily proven:

Proposition 5 (Convexity properties of power laws).

(i) If $q \in(1,2]$, then $h(r)=r^{q}$ is $\lambda$-convex on $[0, R]$ for $\lambda=\inf _{(0, R)} h^{\prime \prime}=q(q-1) R^{q-2}>0$, and thus, uniformly convex on $[0, R]$.

(ii) If $q>2$, then $h(r)=r^{q}$ is $\phi$-uniformly convex on $\mathbb{R}_{+}$, with $\phi(t)=2^{2-q} t^{q-1} / q$. That is

$$
h\left(\frac{r_{1}+r_{2}}{2}\right) \leq \frac{1}{2}\left(h\left(r_{1}\right)+h\left(r_{2}\right)\right)-2^{-q}\left|r_{1}-r_{2}\right|^{q},
$$

for $r_{1}, r_{2} \geq 0$.

4.2. Proof of Theorem 2, In this subsection we prove the following generalization of Theorem 2.

Theorem 3. Let $W(x)=w(|x|)$ be continuously differentiable, bounded from below, and decreasing as a function of $|x|$ in a neighborhood of the origin. Assume moreover that $W$ behaves like the power law $-|x|^{\alpha}, \alpha>2$, near the origin, in the sense that for some $C^{*}>0$ and $R>0$ small enough, $W(r)=-h\left(r^{2}\right)$ satisfies:

- if $\alpha \in(2,4], h$ is $\lambda$-convex on $[0, R]$ with $\lambda=C^{*} R^{\alpha / 2-2}$.

- if $\alpha \in(4, \infty), h$ is $\phi$-uniformly convex on $[0, R]$, with $\phi(t)=C^{*} t^{\alpha / 2-1}$,

and $C^{*}\left|w^{\prime}(r)\right| \leq r^{\alpha-1}$ on $[0, R]$. Then a local minimizer of the interaction energy with respect to $d_{\infty}$ cannot have a $k$-dimensional part for any $1 \leq k \leq N$.

Theorem 2 is a direct consequence of Theorem 3 , thanks to Proposition 5.

We first provide an explicit formula for how the energy changes when perturbing a local minimizer:

Lemma 4. Suppose that $W: \mathbb{R}^{N} \rightarrow(-\infty,+\infty]$ is symmetric, l.s.c. and bounded from below with $W(0)<+\infty$. Let $\mu \in \mathcal{P}\left(\mathbb{R}^{N}\right)$ be a local minimizer of the interaction energy with respect to $d_{\infty}$ and $E[\mu]<+\infty$. Given a connected domain $\Omega \subseteq \operatorname{supp}(\mu)$, a Borel map $\pi: \Omega \rightarrow \Omega$ and a convex decomposition $\mu=m_{1} \mu_{1}+m_{2} \mu_{2}$ with $\operatorname{supp}\left(\mu_{1}\right) \subset \Omega$, we deduce that

$$
E\left[m_{1}\left(\pi \# \mu_{1}\right)+m_{2} \mu_{2}\right]-E\left[m_{1} \mu_{1}+m_{2} \mu_{2}\right]=m_{1}^{2} T\left[\pi \# \mu_{1}, \mu_{1}\right] .
$$

Proof. Since $B$ in (7) is a bilinear form,

$$
\begin{aligned}
E\left[m_{1}\left(\pi \# \mu_{1}\right)+m_{2} \mu_{2}\right]-E & \left.m_{1} \mu_{1}+m_{2} \mu_{2}\right] \\
= & m_{1}^{2} B\left[\pi \# \mu_{1}, \pi \# \mu_{1}\right]+2 m_{1} m_{2} B\left[\pi \# \mu_{1}, \mu_{2}\right] \\
& -m_{1}^{2} B\left[\mu_{1}, \mu_{1}\right]-2 m_{1} m_{2} B\left[\mu_{1}, \mu_{2}\right] .
\end{aligned}
$$

We now use the fact that $\mu$ is local minimizer to express the terms involving $\mu_{2}$ as terms involving only $\mu_{1}$. Proposition 2 implies that the function $V_{\mu}(x)$ is constant on the connected domain $\Omega$ and since $\pi(\Omega) \subset \Omega$ we have:

$$
\int_{\mathbb{R}^{N}} W(\pi(x)-y) d \mu(y)=\int_{\mathbb{R}^{N}} W(x-y) d \mu(y) \quad \forall x \in \Omega .
$$


and therefore, since $\mu=m_{1} \mu_{1}+m_{2} \mu_{2}$,

$$
\begin{aligned}
& m_{1} \int_{\mathbb{R}^{N}} W(\pi(x)-y) d \mu_{1}(y)+m_{2} \int_{\mathbb{R}^{N}} W(\pi(x)-y) d \mu_{2}(y) \\
&=m_{1} \int_{\mathbb{R}^{N}} W(x-y) d \mu_{1}(y)+m_{2} \int_{\mathbb{R}^{N}} W(x-y) d \mu_{2}(y)
\end{aligned}
$$

for all $x \in \Omega$. Since $\operatorname{supp}\left(\mu_{1}\right) \subset \Omega$ we can integrate both sides with respect to $d \mu_{1}(x)$ and obtain, after multiplication by $m_{1}$ :

$$
\begin{aligned}
m_{1}^{2} \int_{\mathbb{R}^{N} \times \mathbb{R}^{N}} W(\pi(x)-y) d \mu_{1}(y) d \mu_{1}(x)+m_{1} m_{2} \int_{\mathbb{R}^{N} \times \mathbb{R}^{N}} W(\pi(x)-y) d \mu_{2}(y) d \mu_{1}(x) \\
\quad=m_{1}^{2} \int_{\mathbb{R}^{N} \times \mathbb{R}^{N}} W(x-y) d \mu_{1}(y) d \mu_{1}(x)+m_{1} m_{2} \int_{\mathbb{R}^{N} \times \mathbb{R}^{N}} W(x-y) d \mu_{2}(y) d \mu_{1}(x)
\end{aligned}
$$

or equivalently, using the $B$-notation,

$$
2 m_{1}^{2} B\left[\mu_{1}, \pi \# \mu_{1}\right]+2 m_{1} m_{2} B\left[\mu_{2}, \pi \# \mu_{1}\right]=2 m_{1}^{2} B\left[\mu_{1}, \mu_{1}\right]+2 m_{1} m_{2} B\left[\mu_{2}, \mu_{1}\right]
$$

and therefore rearranging the terms, we can express the terms involving $\mu_{2}$ in terms of the ones involving only $\mu_{1}$ :

$$
2 m_{1} m_{2}\left[B\left[\pi \# \mu_{1}, \mu_{2}\right]-B\left[\mu_{1}, \mu_{2}\right]\right]=2 m_{1}^{2}\left[B\left[\mu_{1}, \mu_{1}\right]-B\left[\mu_{1}, \pi \# \mu_{1}\right]\right] .
$$

The desired identity (30) is readily obtained by plugging the last equality into (31) reminding the definition of $T[\mu, \nu]$ in Section 2.

Definition 6. Let $1 \leq k \leq N$. We denote by $D_{\varepsilon}^{k}$ the the $k$-dimensional disk of radius $\varepsilon$ :

$$
D_{\varepsilon}^{k}=\left\{\left(x_{1}, \ldots, x_{N}\right): x_{1}^{2}+\ldots+x_{k}^{2} \leq \varepsilon^{2} \text { and } x_{k+1}=\cdots=x_{N}=0\right\},
$$

and by $\nu_{\varepsilon, k} \in \mathcal{P}\left(\mathbb{R}^{N}\right)$ the uniform probability distribution on $D_{\varepsilon}^{k}$ :

$$
\nu_{\varepsilon, k}=\frac{1}{\left|D_{\varepsilon}^{k}\right|} \delta_{D_{\varepsilon}^{k}}
$$

where $\left|D_{\varepsilon}^{k}\right|$ is the Lebesgue measure of dimension $k$ of $D_{\varepsilon}^{k}$, that is $\left|D_{\varepsilon}^{k}\right|=\sigma_{k} \varepsilon^{k}$ where $\sigma_{k}$ the area of the unit $k$-dimensional ball. $\nu_{\varepsilon, k}$ then satisfies

$$
\int_{\mathbb{R}^{N}} \psi(x) d \nu_{\varepsilon, k}(x)=\frac{1}{\left|D_{\varepsilon}^{k}\right|} \int_{x_{1}^{2}+\ldots+x_{k}^{2} \leq \varepsilon^{2}} \psi\left(x_{1}, \ldots, x_{k}, 0, \ldots, 0\right) d x_{1} \ldots d x_{k}
$$

for all $\psi \in C^{0}\left(\mathbb{R}^{N}\right)$.

The following Lemma combined with Lemma 4 shows that if a flat $k$-dimensional disk is contained in the support of a local minimizer, then the energy can be reduced by concentrating all the mass contained in the disk into a single point. As a consequence the support of a local minimizer cannot contain a flat $k$-dimensional disk.

Lemma 5. Suppose that $W(x)=-h\left(|x|^{2}\right)$ satisfies the assumptions of Theorem 3 . Then, there exists $c_{k, \alpha}>0$ such that for $\varepsilon$ small enough,

$$
T\left[\delta_{0}, \nu_{\varepsilon, k}\right]=B\left[\delta_{0}, \delta_{0}\right]-2 B\left[\delta_{0}, \nu_{\varepsilon, k}\right]+B\left[\nu_{\varepsilon, k}, \nu_{\varepsilon, k}\right] \leq-c_{k, \alpha} \varepsilon^{\alpha} .
$$


Proof. Since $W$ is bounded from below and $W(0)<+\infty$, we can assume without loss of generality that $W(0)=0$ by adding to $W$ a suitable constant. Then, the first term $B\left[\delta_{0}, \delta_{0}\right]$ is equal to zero. Symmetrizing the integral involved in second term we obtain:

$$
\begin{aligned}
B\left[\delta_{0}, \nu_{\varepsilon, k}\right]=-\frac{1}{2} \int_{\mathbb{R}^{N}} h\left(|y|^{2}\right) d \nu_{\varepsilon, k}(y) & =-\frac{1}{2} \int_{\mathbb{R}^{N} \times \mathbb{R}^{N}} h\left(|y|^{2}\right) d \nu_{\varepsilon, k}(x) d \nu_{\varepsilon, k}(y) \\
& =-\frac{1}{4} \int_{\mathbb{R}^{N} \times \mathbb{R}^{N}}\left[h\left(|x|^{2}\right)+h\left(|y|^{2}\right)\right] d \nu_{\varepsilon, k}(x) d \nu_{\varepsilon, k}(y) .
\end{aligned}
$$

Since $\nu_{\varepsilon, k}(-y)=\nu_{\varepsilon, k}(y)$, we can also symmetrize the third term and obtain:

$$
\begin{aligned}
B\left[\nu_{\varepsilon, k}, \nu_{\varepsilon, k}\right] & =-\frac{1}{2} \int_{\mathbb{R}^{N} \times \mathbb{R}^{N}} h\left(|x-y|^{2}\right) d \nu_{\varepsilon, k}(x) d \nu_{\varepsilon, k}(y) \\
& =-\frac{1}{4} \int_{\mathbb{R}^{N} \times \mathbb{R}^{N}}\left[h\left(|x-y|^{2}\right)+h\left(|x+y|^{2}\right)\right] d \nu_{\varepsilon, k}(x) d \nu_{\varepsilon, k}(y) .
\end{aligned}
$$

Combining the three terms we find

$$
T\left[\delta_{0}, \nu_{\varepsilon, k}\right]=\frac{1}{2} \int_{\mathbb{R}^{N} \times \mathbb{R}^{N}} A(x, y) d \nu_{\varepsilon, k}(x) d \nu_{\varepsilon, k}(y)
$$

with

$$
A(x, y):=h\left(|x|^{2}\right)+h\left(|y|^{2}\right)-\frac{h\left(|x-y|^{2}\right)+h\left(|x+y|^{2}\right)}{2} .
$$

Under the assumptions of Theorem $2, h$ is convex on $\left(0,2 \varepsilon^{2}\right)$ and since $h(0)=0$, we deduce

$$
h\left(r_{i}^{2}\right) \leq \frac{r_{i}^{2}}{r_{1}^{2}+r_{2}^{2}} h\left(r_{1}^{2}+r_{2}^{2}\right),
$$

for $r_{i} \geq 0, i=1,2$. Using the above inequalities for $i=1,2$ we get

$$
h\left(|x|^{2}\right)+h\left(|y|^{2}\right) \leq h\left(|x|^{2}+|y|^{2}\right)=h\left(\frac{1}{2}|x+y|^{2}+\frac{1}{2}|x-y|^{2}\right) .
$$

In the rest of this Lemma, $C$ will denote some generic constant that will change from step to step. For $\alpha \in(2,4], h$ is $\lambda$-convex on $\left(0,2 \varepsilon^{2}\right)$ with $\lambda=C \varepsilon^{\alpha-4}$, so that plugging into (28), we obtain

$$
h\left(|x|^{2}\right)+h\left(|y|^{2}\right) \leq \frac{1}{2} h\left(|x+y|^{2}\right)+\frac{1}{2} h\left(|x-y|^{2}\right)-C \varepsilon^{\alpha-4}(x \cdot y)^{2} .
$$

Combining (32) and (33) we get:

$$
\begin{aligned}
T\left[\delta_{0}, \nu_{\varepsilon, k}\right] & \leq-C \varepsilon^{\alpha-4} \int_{\mathbb{R}^{N} \times \mathbb{R}^{N}}(x \cdot y)^{2} d \nu_{\varepsilon, k}(x) d \nu_{\varepsilon, k}(y) \\
& =-C \varepsilon^{\alpha} \int_{\mathbb{R}^{N} \times \mathbb{R}^{N}}(x \cdot y)^{2} d \nu_{1, k}(x) d \nu_{1, k}(y) .
\end{aligned}
$$

For $\alpha \geq 4, h$ is $\phi$-convex with $\phi(t)=C t^{\alpha / 2-1}$, so that plugging into $[29$, we obtain

$$
h\left(|x|^{2}\right)+h\left(|y|^{2}\right) \leq \frac{1}{2} h\left(|x+y|^{2}\right)+\frac{1}{2} h\left(|x-y|^{2}\right)-C|x \cdot y|^{\alpha / 2} .
$$

Combining 32 and 34 we get:

$$
\begin{aligned}
T\left[\delta_{0}, \nu_{\varepsilon, k}\right] & \leq-C \int_{\mathbb{R}^{N} \times \mathbb{R}^{N}}|x \cdot y|^{\alpha / 2} d \nu_{\varepsilon, k}(x) d \nu_{\varepsilon, k}(y) \\
& =-C \varepsilon^{\alpha} \int_{\mathbb{R}^{N} \times \mathbb{R}^{N}}|x \cdot y|^{\alpha / 2} d \nu_{1, k}(x) d \nu_{1, k}(y) .
\end{aligned}
$$


The last Lemma combined to Lemma 4 shows that the support of a local minimizer cannot contain a flat $k$-dimensional disk of radius $\epsilon$. To conclude the proof of Theorem 3, we need to introduce some differential geometry tools. Let $R>0$, and $g: D_{R}^{k} \rightarrow \mathbb{R}^{N-k}$ a $C^{2}$-function such that $g(0)=0, \nabla g(0)=0$. We define the parameterisation $P_{g}$ of the graph of $g$ as follows:

$$
\begin{array}{ccc}
P_{g}: D_{R}^{k} & \longrightarrow & \mathbb{R}^{N}, \\
\left(x^{\prime}, 0\right) & \mapsto & \left(x^{\prime}, g\left(x^{\prime}\right)\right)
\end{array}
$$

where $x^{\prime}=\left(x_{1}, \ldots, x_{k}\right)$ stands for the $k$ first coordinates. Let us remark that classical differential geometry implies that any $C^{2}$-manifold can be locally parameterized by such graphs by choosing conveniently the axis and reordering of variables. Moreover, this can be done in such a way that the volume element of the graph $J_{g}$ is as close to the unit volume element of the flat tangent space by taking $R$ small enough, see [29]. More precisely, there exists a constant $C_{g}$ depending only on the second derivatives of $g$ on $D_{R}^{k}$ such that

$$
\left\|J_{g}-1\right\|_{L^{\infty}\left(D_{\varepsilon}^{k}\right)} \leq C_{g} \varepsilon
$$

for $0<\varepsilon<R$ small enough.

Lemma 6. If $W$ satisfies the assumptions of Theorem 3, and $g \in C^{2}\left(D_{R}^{k}, \mathbb{R}^{N-k}\right)$ satisfies $g(0)=0$, $\nabla g(0)=0$, then for $\varepsilon>0$ small enough,

$$
T\left[\delta_{0}, P_{g} \# \nu_{\varepsilon, k}\right]-T\left[\delta_{0}, \nu_{\varepsilon, k}\right] \leq \frac{2^{\alpha-1} \varepsilon^{\alpha}}{C^{*}}\|\nabla g\|_{L^{\infty}\left(D_{\varepsilon}^{k}\right)} .
$$

Proof. Note that by continuity for $\varepsilon>0$ small enough, we have $\|\nabla g\|_{L^{\infty}\left(D_{\varepsilon}^{k}\right)} \leq 1$. We first point out that

$$
T\left[\delta_{0}, P_{g} \# \nu_{\varepsilon, k}\right]-T\left[\delta_{0}, \nu_{\varepsilon, k}\right]=\int_{\mathbb{R}^{N} \times \mathbb{R}^{N}} A(x, y) d \nu_{\varepsilon, k}(x) d \nu_{\varepsilon, k}(y)
$$

with

$$
A(x, y)=\frac{w\left(\left|P_{g}(x)-P_{g}(y)\right|\right)-w(|x-y|)}{2}-\left[w\left(\left|P_{g}(x)\right|\right)-w(|x|)\right] .
$$

Thanks to the definition of the parameterisation $P_{g},\left|P_{g}(x)-P_{g}(y)\right| \geq|x-y|$. Moreover since $w$ is decreasing in a neighborhood of the 0 , the first term in $A(x, y)$ is negative for $\max (|x|,|y|)<\varepsilon$ small enough. To estimate the second term, we use the mean value theorem for $g$ around $x^{\prime}=0$, remembering that $g(0)=0$ :

$$
\left|P_{g}(x)-\left(x^{\prime}, 0\right)\right|=\left|g\left(x^{\prime}\right)-g(0)\right| \leq \varepsilon\|\nabla g\|_{L^{\infty}\left(D_{\varepsilon}^{k}\right)}
$$

since $C^{*}\left|w^{\prime}(r)\right| \leq r^{\alpha-1}$, we conclude

$$
w\left(\left|P_{g}(x)\right|\right)-w(|x|) \leq\left\|w^{\prime}\right\|_{L^{\infty}([0,2 \varepsilon])} \varepsilon\|\nabla g\|_{L^{\infty}\left(D_{\varepsilon}^{k}\right)} \leq \frac{2^{\alpha-1} \varepsilon^{\alpha}}{C^{*}}\|\nabla g\|_{L^{\infty}\left(D_{\varepsilon}^{k}\right)} .
$$

Proof of the Theorem 2. Assume that $\mu$ is a local minimizer of $E$ in $d_{\infty}$ and that it has a regular $k$-dimensional part in the sense of Definition 4. Let $\mathcal{M}$ be the $C^{1}$-submanifold on which this component is supported, and $f$ be the density on $\mathcal{M}$ of this component. Due to assumption (26) there exists $x_{0} \in \mathcal{M}, c, \kappa>0$ such that

$$
f(x) \geq c, \quad \forall x \in \mathcal{M} \cap B\left(x_{0}, \kappa\right) .
$$

As discussed above and without loss of generality, we can assume that $x_{0}=0$ and that $\mathcal{M}$ is locally the graph of a $C^{2}$-function $g: D_{R}^{k} \rightarrow \mathbb{R}^{N-k}$, for some $\kappa>R>0$, such that $g(0)=0, \nabla g(0)=0$. 
Let $P_{g}$ be the parameterisation defined in (35). Note that for $\varepsilon \leq R, \mu_{1}^{\varepsilon}:=P_{g} \# \nu_{\varepsilon, k} \in \mathcal{P}\left(\mathbb{R}^{N}\right)$ is absolutely continuous with respect to the volume element on $\mathcal{M}$ with a density denoted still by $\mu_{1}^{\varepsilon}$ satisfying

$$
\left\|\mu_{1}^{\varepsilon}\right\|_{L^{\infty}(\mathcal{M}, d \sigma)} \leq \frac{1}{\left|D_{\varepsilon}^{k}\right| I_{\varepsilon}} \leq \frac{1}{\left|D_{\varepsilon}^{k}\right|\left(1-C_{g} \varepsilon\right)}, \quad \text { with } I_{\varepsilon}=\inf _{x \in \bar{D}_{\varepsilon}^{k}} J_{g}(x) .
$$

where we used (36). Therefore, choosing $m_{1}=\frac{c}{2}\left|D_{\varepsilon}^{k}\right|\left(1-C_{g} \varepsilon\right)$, then $f(x)>m_{1} \mu_{1}^{\varepsilon}$ on $x \in D_{\varepsilon}^{k}$, and we can decompose $\mu$ as a convex combination

$$
\mu=m_{1} \mu_{1}^{\varepsilon}+m_{2} \mu_{2}^{\varepsilon},
$$

where $\mu_{2}^{\varepsilon} \in \mathcal{P}\left(\mathbb{R}^{N}\right)$.

We are going to send now all mass from $\mu_{1}^{\varepsilon}$ to a Dirac Delta at $x_{0}=0$. Let us define $\pi: \mathbb{R}^{N} \longrightarrow$ $\mathbb{R}^{N}$ by $\pi \equiv 0$ and $\mu^{\varepsilon}:=m_{1} \pi \# \mu_{1}^{\varepsilon}+m_{2} \mu_{2}^{\varepsilon}$, then $\pi \# \mu_{1}^{\varepsilon}=\delta_{0}$. Moreover, $\mu^{\varepsilon}$ is a small perturbation of $\mu$ in $d_{\infty}$ :

$$
d_{\infty}\left(\mu, \mu^{\varepsilon}\right) \leq \varepsilon\left(1+\|\nabla g\|_{L^{\infty}\left(D_{\varepsilon}^{k}\right)}\right) .
$$

To check this just take a map $\mathcal{T}$ in Definition 9 such that $\mathcal{T}(x)=x$ for all $x \in \mathcal{M} / P_{g}\left(D_{\varepsilon}^{k}\right)$ and such that $\mathcal{T}(x)=0$ for $x \in P_{g}\left(D_{\varepsilon}^{k}\right)$. Thus, the maximum displacement produced by the transport map $\mathcal{T}$ is bounded by the maximum of $\left|P_{g}(x)\right|$ for $x \in P_{g}\left(D_{\varepsilon}^{k}\right)$ leading to (37) using that $g(0)=0$ and the mean value theorem.

Since $\mu_{1}^{\varepsilon}$ has a connected support that contains $\pi\left(\operatorname{supp}\left(\mu_{1}^{\varepsilon}\right)\right)=\{0\}$, we can apply Lemma 4 to get:

$$
\begin{aligned}
E\left[\mu^{\varepsilon}\right]-E[\mu] & =m_{1}^{2} T\left[\pi \# \mu_{1}^{\varepsilon}, \mu_{1}^{\varepsilon}\right] \\
& =m_{1}^{2} T\left[\pi \# \mu_{1}^{\varepsilon}, \nu_{\varepsilon, k}\right]+m_{1}^{2}\left(T\left[\pi \# \mu_{1}^{\varepsilon}, \mu_{1}^{\varepsilon}\right]-T\left[\pi \# \mu_{1}^{\varepsilon}, \nu_{\varepsilon, k}\right]\right)
\end{aligned}
$$

Since $\pi \# \mu_{1}^{\varepsilon}=\delta_{0}$, we can use Lemma 5 to estimate the first term, and since moreover $\mu_{1}^{\varepsilon}=P_{g} \# \nu_{\varepsilon, k}$, we can use Lemma 6 to estimate the last two terms, so that we finally conclude

$$
E\left[\mu^{\varepsilon}\right]-E[\mu] \leq m_{1}^{2}\left[-c_{k, \alpha} \varepsilon^{\alpha}+C \varepsilon^{\alpha}\|\nabla g\|_{L^{\infty}\left(D_{\varepsilon}^{k}\right)}\right] .
$$

Since $g \in C^{1}\left(D_{R}^{k}\right)$ and $\nabla g(0)=0$ imply that $\|\nabla g\|_{L^{\infty}\left(D_{\varepsilon}^{k}\right)} \rightarrow 0$ as $\varepsilon \rightarrow 0$, thus if $\varepsilon>0$ is small enough, $E\left[\mu^{\varepsilon}\right]-E[\mu]<0$.

Thus, $\mu^{\varepsilon}$ is a better competitor in the minimization of $E$ for $\varepsilon$ arbitrary small. This leads to a contradiction with the fact that $\mu$ is a local minimizer of $E$ showing Theorem 2 ,

\section{Euler-Lagrange APPROACH TO STUdy LOCAL Minimizers IN THE $d_{2}$-TOPOLOGY}

So far we have used transport plans to build perturbed measures. This enabled us to study local minimizers of the interaction energy with respect to the $d_{\infty}$-topology. To study local minimizers with respect to the $d_{2}$-topology it is actually possible to use a more classical Euler-Lagrange approach as we will present in this section. The Euler-Lagrange conditions that we will derive were formally obtained in [4] by perturbing densities inside and outside their support. Here, we provide a fully rigorous proof in the case of probability measures endowed with the distance $d_{2}$.

Theorem 4. Given an interaction potential $W$ satisfying $(\mathrm{H} 1)-(\mathrm{H} 2)$. Let us consider $\mu \in \mathcal{P}_{2}\left(\mathbb{R}^{N}\right)$ a local minimizer of $E$ with respect to $d_{2}$ such that $E[\mu]<\infty$. Then,

(i) $(W * \mu)(x)=2 E[\mu] \mu$-a.e.

(ii) $(W * \mu)(x) \leq 2 E[\mu]$ for all $x \in \operatorname{supp}(\mu)$.

(iii) $(W * \mu)(x) \geq 2 E[\mu]$ for a.e. $x \in \mathbb{R}^{N}$. 
Proof. As usual, we assume that $W \geq 0$ without loss of generality. Lemma 2 implies that $W * \mu$ is well defined, lower semicontinuous, and non-negative.

In order to prove the first two items, let us choose $\varphi \in C_{0}^{\infty}\left(\mathbb{R}^{N}\right)$ to define

$$
\nu=\left(\varphi-\int_{\mathbb{R}^{N}} \varphi d \mu\right) \mu:=a(x) \mu
$$

and $\mu_{\epsilon}=\mu+\epsilon \nu=(1+\epsilon a(x)) \mu$ with $\epsilon>0$ to be specified. It is clear that $\mu_{\epsilon}\left(\mathbb{R}^{N}\right)=1$ since $a(x)$ has zero integral with respect to $\mu$. Moreover, since $a(x) \geq-2\|\varphi\|_{L^{\infty}}$ then $\mu_{\epsilon} \geq 0$ for $\epsilon<\frac{1}{2\|\varphi\|_{L^{\infty}}}=\epsilon_{\varphi}$. Therefore, $\mu_{\epsilon} \in \mathcal{P}\left(\mathbb{R}^{N}\right)$ for all $\epsilon<\epsilon_{\varphi}$. It is easy to check that $\mu_{\epsilon} \in \mathcal{P}_{2}\left(\mathbb{R}^{N}\right)$, that $\mu_{\epsilon} \rightarrow \mu$ weakly-* as measures, and

$$
\int_{\mathbb{R}^{N}}|x|^{2} d \mu_{\epsilon} \rightarrow \int_{\mathbb{R}^{N}}|x|^{2} d \mu
$$

In fact, since $\epsilon a(x)$ converges uniformly to 0 , these claims follow by dominated convergence theorem. Therefore, we conclude that

$$
d_{2}\left(\mu_{\epsilon}, \mu\right) \rightarrow 0 \quad \text { as } \quad \epsilon \rightarrow 0 .
$$

Note that it is not true that $d_{\infty}\left(\mu_{\epsilon}, \mu\right) \rightarrow 0$ as $\epsilon \rightarrow 0$ since for localized test functions $\varphi$ in subsets of the $\operatorname{supp}(\mu)$, we are always forced to move mass in $\operatorname{supp}(\mu)$ for a fixed distance not depending on $\epsilon$.

Now, since $\mu$ is a local minimizer in $d_{2}$ then $E\left[\mu_{\epsilon}\right] \geq E[\mu]$ for $\epsilon$ small enough. Moreover, since $\mu$ has finite energy, then $E\left[\mu_{\epsilon}\right]<\infty$ and we can expand it as

$$
\frac{E\left[\mu_{\epsilon}\right]-E[\mu]}{\epsilon}=\iint_{\mathbb{R}^{N} \times \mathbb{R}^{N}} W(x-y) d \nu(x) d \mu(y)+\frac{\epsilon}{2} \iint_{\mathbb{R}^{N} \times \mathbb{R}^{N}} W(x-y) d \nu(x) d \nu(y) \geq 0,
$$

with both integral terms well-defined. As $\epsilon \rightarrow 0$, we easily get

$$
\iint_{\mathbb{R}^{N} \times \mathbb{R}^{N}} W(x-y) d \nu(x) d \mu(y) \geq 0
$$

or equivalently,

$$
\int \varphi[(W * \mu)(x)-2 E[\mu]] d \mu(x) \geq 0
$$

for all $\varphi \in C_{0}^{\infty}\left(\mathbb{R}^{N}\right)$. Since one can take either $\varphi$ or $-\varphi$ as test functions, we deduce

$$
\int \varphi[(W * \mu)(x)-2 E[\mu]] d \mu(x)=0
$$

for all $\varphi \in C_{0}^{\infty}\left(\mathbb{R}^{N}\right)$, and thus (i) is satisfied a.e. $\mu$.

Let us now prove (ii). Take $x \in \operatorname{supp}(\mu)$ then there exists $\left\{x_{n}\right\}_{n \in \mathbb{N}} \rightarrow x$ with $x_{n} \in \operatorname{supp}(\mu)$, such that $(W * \mu)\left(x_{n}\right)=2 E[\mu]$. The existence of such a sequence is ensured since $\mu(B(x, \epsilon))>0$ for all $\epsilon>0$ by definition of the support of $\mu$. Then, by lower semicontinuity of $W * \mu$ we get

$$
(W * \mu)(x) \leq \liminf _{n \rightarrow \infty}(W * \mu)\left(x_{n}\right)=2 E[\mu] .
$$

and then (ii) is satisfied.

In order to show (iii), we consider different variations to the ones constructed above. Take $\psi \in \mathcal{C}_{0}^{\infty}\left(\mathbb{R}^{N}\right), \psi \geq 0$ and then take

$$
\nu=\psi-\left(\int_{\mathbb{R}^{N}} \psi d x\right) \mu .
$$

Again, defining $\mu_{\epsilon}=\mu+\epsilon \nu$, then it verifies $\mu_{\epsilon}\left(\mathbb{R}^{N}\right)=1$ and if $\epsilon<1 / \int \psi d x$ then $\mu_{\epsilon} \geq 0$. Let us remark that this cannot be done for a changing sign test function $\psi$. As previously, it is easy to check that

$$
d_{2}\left(\mu_{\epsilon}, \mu\right) \rightarrow 0 \quad \text { as } \quad \epsilon \rightarrow 0
$$


note that it is not true that $d_{\infty}\left(\mu_{\epsilon}, \mu\right) \rightarrow 0$ as $\epsilon \rightarrow 0$ since we always need to transport some mass from outside the support of $\mu$ to $\operatorname{supp}(\mu)$.

Proceeding similarly as in point (i), we get

$$
\iint_{\mathbb{R}^{N} \times \mathbb{R}^{N}} W(x-y) d \nu(y) d \mu(x) \geq 0
$$

taking $\epsilon \rightarrow 0$ in $E\left[\mu_{\epsilon}\right] \geq E[\mu]$. Therefore, we conclude that

$$
\int_{\mathbb{R}^{N}}((W * \mu)(x)-2 E[\mu]) \psi d x \geq 0
$$

for all $\psi \in C_{0}^{\infty}\left(\mathbb{R}^{N}\right), \psi \geq 0$. This readily implies (iii).

Remark 4. Note that putting together (i), (ii), and (iii) in previous theorem, we conclude that

$$
\left\{\begin{array}{llll}
(W * \mu)(x) & =2 E[\mu] & \text { for a.e. } & x \in \operatorname{supp}(\mu) \\
(W * \mu)(x) & \geq 2 E[\mu] & \text { for a.e. } & x \in \mathbb{R}^{N} \backslash \operatorname{supp}(\mu) .
\end{array}\right.
$$

if $\mu$ is absolutely continuous with respect to the Lebesgue measure. These two properties are the Euler-Lagrange conditions that were found for densities in [4].

Remark 5. Let us now clarify the differences between local minimizers in the $d_{2}$-topology and local minimizers in the $d_{\infty}$-topology. Following [17], let consider as an example the interaction potential $W(x):=-x^{2}+\frac{x^{4}}{2}$ in one dimension. Then,

$$
\rho_{m}=m \delta_{0}+(1-m) \delta_{1}
$$

is a critical point of the interaction energy for any $m \in[0,1]$. Theorem 3.1 in [17] shows that the measure $\rho_{m}$ is a local minimizer in the $d_{\infty}$-topology as soon as $m \in(1 / 3,2 / 3)$. Indeed, what is proven is the stronger statement that $\rho_{m}$ is locally asymptotically stable for the aggregation equation (6) with respect to any perturbation in the $d_{\infty}$-topology. However, $E\left(\rho_{m}\right)=\frac{1}{2}\left(m-\frac{1}{2}\right)^{2}-\frac{1}{8}$, so that $\rho_{1 / 2}$ only can be a local minimizer of the energy in the $d_{2}$-topology (and one can prove that it actually is). This shows that the set of local minimizers with respect to the $d_{2}$-topology is strictly contained in the set of local minimizers with respect to the $d_{\infty}$-topology. Moreover, numerical simulations suggest that, for $m \in(1 / 3,2 / 3), \rho_{m}$ is actually stable (although not asymptotically stable) with respect to small $d_{2}$-perturbations. As a consequence, when using a gradient flow approach to compute numerically minimizers of the interaction energy via particles, one obtains $d_{\infty}$-local minimizers which typically are not $d_{2}$-local minimizers (see e.g. Fig 2 of [17]).

\section{Numerical Experiments}

In this section we conduct a numerical investigation of the local minimizers of the discrete interaction energy (2) with high number of particles. The gradient flow of (2) is given by the system of ODEs:

$$
\dot{X}_{i}=-\sum_{\substack{j=1 \\ j \neq i}}^{n} m_{j} \nabla W\left(X_{i}-X_{j}\right)
$$

In order to efficiently find local minimizers of (2), we solve (38) by an explicit Euler scheme with an adaptive time step chosen as the largest possible such that the discrete energy (2) decreases. This scheme is nothing else than a gradient descent method for the discrete energy (2). Although this method might not be accurate enough for the dynamics, it is efficient to find local minimizers of the discrete energy. In stiffer situations an explicit Runge-Kutta method is used instead. These methods are essentially the ones proposed in [36, 35. 


\begin{tabular}{|c|c|c|c|c|}
\hline & $\operatorname{Dim}=0$ & $\operatorname{Dim}=1$ & $\operatorname{Dim}=2$ & $\operatorname{Dim}=3$ \\
\hline$\alpha=2.5$ & (a) & & & \\
\hline 更 & & $?$ & $\begin{array}{l}\text { (b) } \\
\nabla_{\Delta} \\
\Delta\end{array}$ & (c) \\
\hline$\alpha=0.5$ & & & (d) & (e) \\
\hline$\alpha=-0.5$ & & & & (f) \\
\hline
\end{tabular}

TABle 2. Minimizers of $E_{W}^{n}$ in $\mathbb{R}^{3}$ for various power-law potentials with $n=2,500$.

The results of these simulations in two dimensions with power-law potentials were presented in the introduction, see Table 1. In Subsection 6.1 we discuss similar computations in three dimensions. We also provide numerical experiments suggesting that for some potentials, there are local minimizers of the interaction energy with mixed dimensionality, that is, local minimizers that are the sum of measures whose support have different Hausdorff dimension.

In Subsection 6.2 we show how our numerical results can be further understood by using the results from [23, 36, 3], where a careful stability analysis of ring solution (in 2D) and spherical shell solution (in 3D) was conducted. We also show how this stability analysis connects to the analytical results presented in this paper.

6.1. Numerical experiments in 3D. We first compute numerically local minimizers of $E_{W}^{n}$ where $W$ is the power-law potential defined by (5). Recall that $\Delta W(x) \sim-1 /|x|^{\beta}$ with $\beta=2-\alpha$ as $x \rightarrow 0$. The computations are performed with $n=2,500$ particles. The results are shown in Table 2 and are discussed below: 
- Subfigure (a): $\alpha=2.5$ and $\gamma=5$. The support of the minimizer has zero Hausdorff dimension in agreement with Theorem 2. Actually, in this particular case it is supported on 4 points forming a tetrahedron.

- Subfigure (b) and (c): the two potentials have the same behavior at the origin, $\alpha=1.25$, but different attractive long range behavior $(\gamma=15$ and $\gamma=1.4$ respectively). Theorem 1 shows that the Hausdorff dimension of the support must be greater or equal to $\beta=2-\alpha=0.75$. Numerically, we observe that the local minimizer for the first example has a two-dimensional support and the minimizer for the second example has a three-dimensional support. We did not choose the value $\alpha=1.5$ because we were not able to obtain a change of dimensionality of the stable steady states varying $\gamma>\alpha$. Note that $\alpha=1.5$ is always above the instability curve for radial perturbations which meets line $\alpha=\gamma$ at the point $(\sqrt{2}, \sqrt{2})$. See Figure 4 and Subsection 6.2 for more details.

- Subfigure (d) and (e): the two potentials have the same behavior at the origin, $\alpha=0.5$, but different attractive long range behavior $(\gamma=23$ and $\gamma=1.4$ respectively). Theorem 1 shows that the Hausdorff dimension of the support must be greater or equal to $\beta=2-\alpha=1.5$. Numerically, we observe that the local minimizer for the first example has a two-dimensional support and the local minimizer for the second example has a three-dimensional support.

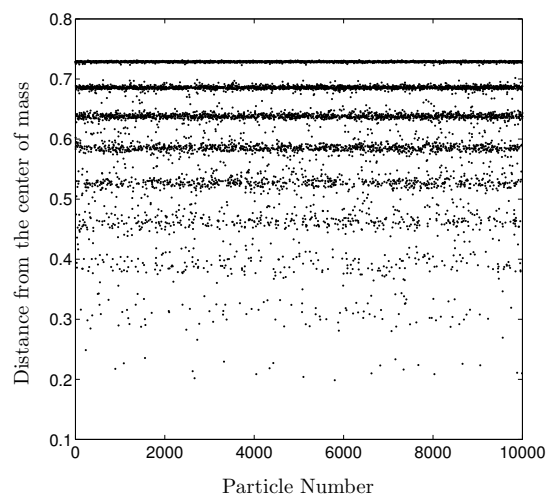

FiguRE 1. Distances of the particles from the center of mass for the power law potential with $\alpha=-0.5, \gamma=5$ in 3D. Case (f) in Subsection 6.1 in Table 2 with $n=10,000$.

- Subfigure (f): $\alpha=-0.5$ and $\gamma=5$. Theorem 1 proves that the Hausdorff dimension of the support must be greater than $\beta=2-\alpha=2.5$, which can also be observed numerically. In Figure 1, we have represented the radius of particles to the center of mass. The particles seem to organize into successive two dimensional layers. Such lattices were also observed in [22], and it is related to the finite number of particles used in the simulations.

Notice that we were not able to find examples of interaction potentials leading numerically to a local minimizer with one dimensional support. We could however observe such situations with an additional asymmetric confining potentials, we thus believe it should be possible to produce such cases.

A natural question following Tables 1 and 2 is whether it is possible to produce local minimizers that are a sum of two measures whose support have different Hausdorff dimensions. A possible candidate was already observed in [36]. Here, we analyze it more carefully with much larger number of particles. From our simulations, it seems that the interaction potential $W(x)=w(|x|)$ with $w$ 
defined by

$$
-w^{\prime}(r)=\tanh ((1-r) a)+b, \quad a=5, \quad b=0.5,
$$

leads numerically to a local minimizer consisting in a ball (Hausdorff dimension three) inside a spherical shell (Hausdorff dimension two), see Figure 2.
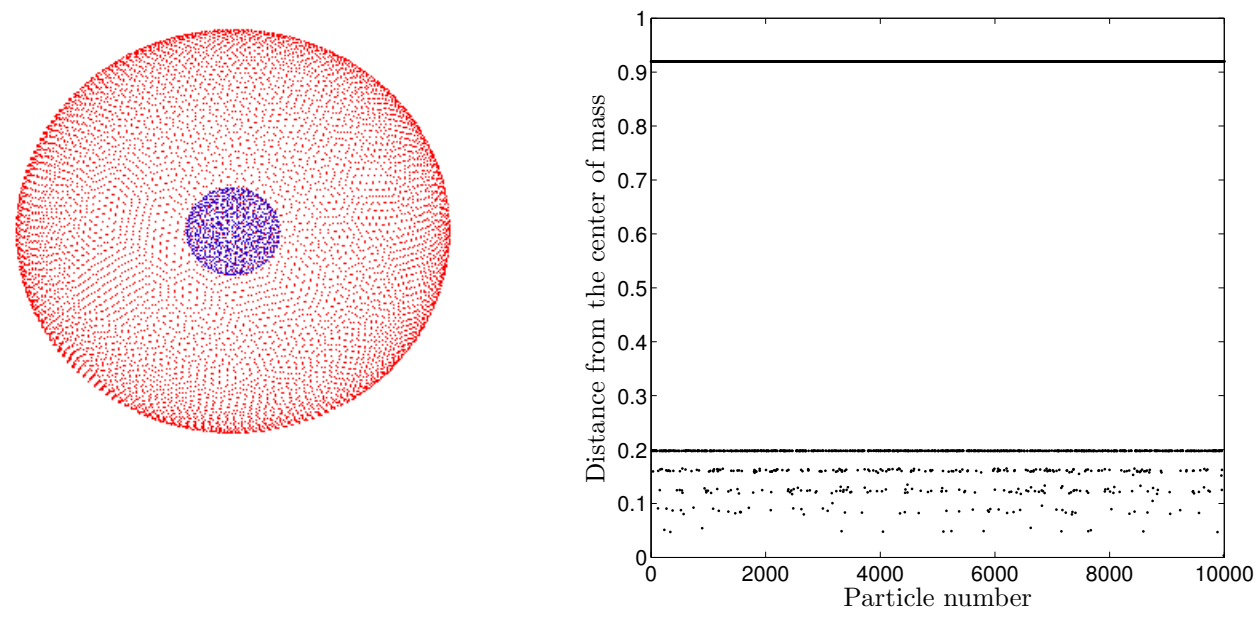

Figure 2. Left: Local minimizer in $3 \mathrm{D}$ with $n=10,000$. Right: Distance of the particles from the center of mass.

The distance of each particle to the center of mass is displayed on the right part of Figure 2 The inner ball appears to be composed of five equally spaced layers of particles. This is most likely due to the fact that particles are organized into a lattice configuration, and therefore the distances between the particles and the origin do not form a continuum. It is instructive to compare the distribution of the radius of the particles in the right subplot of Figure 2 with the one in Figure 1 for the case of an approximated local minimizer with three dimensional support, i.e., Case (f) of Table 2. Although Theorem 1 guarantees that the support of the local minimizer corresponding to Figure 1 has Hausdorff dimension greater or equal to 2.5, we can also observe that particles arrange themselves in layers. Notice that in dimension $\mathrm{N}=2$, such artifacts also appear in simulations using a finite number of particles, see Figure 4 in [22].

6.2. Relationship with previous works on ring and shell solutions. An important characteristic of the analysis performed in the main theorems of this work is that we do not assume a specific shape on the local minimizers. If on the contrary, one is interested by the special case of delta ring minimizers (in 2D), or spherical shell minimizers (in 3D), perturbative methods provide more detailed results.

In [23] the local stability of discrete ring solutions, made of $N$-particle equally distributed in a circle, was studied for the $N$-particle system (38). The authors considered the power law interaction potentials (5), and led a formal linear stability analysis for the continuum ring solution as steady state of the aggregation equation (6) by taking $N \rightarrow \infty$. Those predictions were then confirmed numerically. They could not obtain nonlinear stability of the ring solution particularly because there is no spectral gap as $N \rightarrow \infty$, i.e, the largest negative eigenvalue tends to 0 when $N \rightarrow \infty$. In [3], the nonlinear stability of the ring solutions was proved for radial perturbations, corroborating some 
of the formal results of [23], together with the instability due to fattening in the complementary set of parameters.

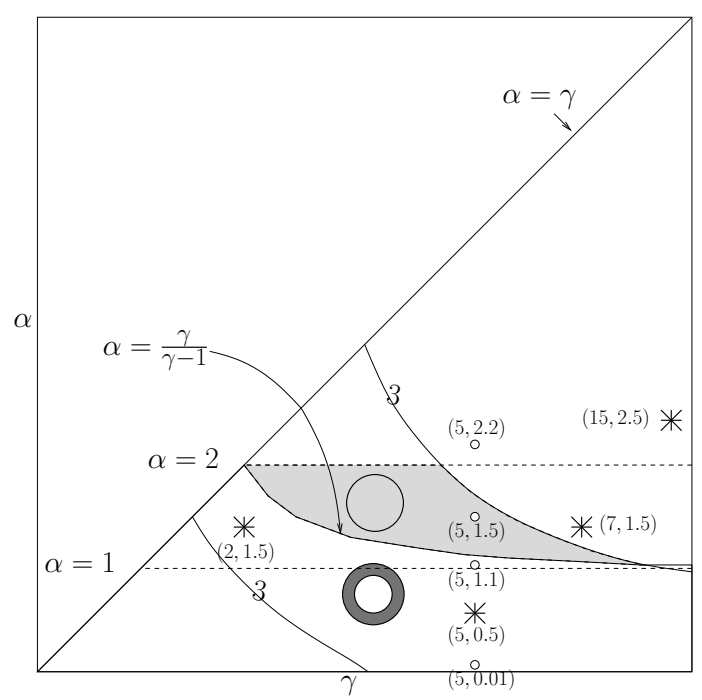

Figure 3. Sketch of all the computed cases in dimension $N=2$. The parameters $(\gamma, \alpha)$ used in Table 1 are marked with $*$, while those used in Table 3 are marked with $\circ$. Notice that $\alpha<\gamma$ is necessary for the interaction potential to be confining. In dark gray is represented the set of parameters such that a delta ring could be a local minimizer.

We have represented this set of parameters in Figure 3, as well as all the parameters used in the two dimensional numerical simulations of this article (Tables 1 and 3). As the caricature presented in Table 3 shows, crossing the lower border of this set, curve $\alpha=\gamma /(\gamma-1)$, leads to a "fattening" of the delta ring, that is to minimizers with dimensionality 2, see [23, 3]. On the other hand, crossing its upper border, given by the curve marked with 3, does not modify the dimensionality of the stable steady states as long as $\alpha<2$ (they remain one dimensional), but leads to a "shape" instability towards a triangular configuration that breaks the ring into 3 connected one dimensional components as in case (b) of Table 1 .

Finally, if $\alpha>2$, local minimizers become of dimensionality 0 , as predicted by Theorem 2 , whereas if $\alpha<1$, all the minimizers are of dimensionality 2, as shown by Theorem 1 .

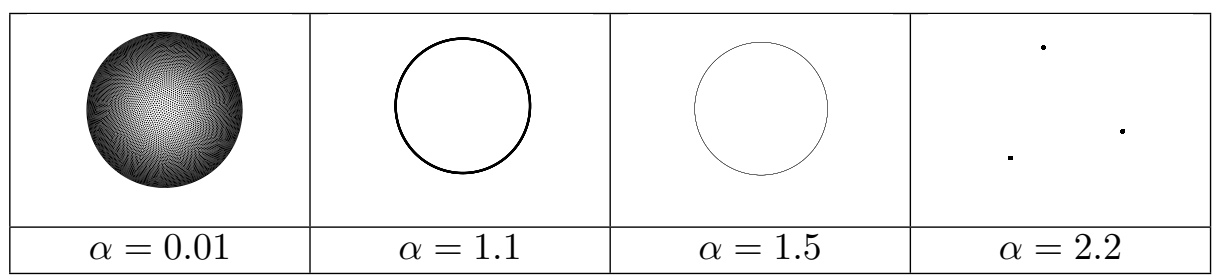

TABLE 3. Evolution of local minimizers when $\alpha>0$ increases, while $\gamma=5$ remains constant. The computations were done with $n=10,000$ particles.

In three dimensions, a linear stability analysis of discrete spherical shell solutions is also possible but it leads to more cumbersome instability curves, see [36, 35. Again, the results in [3] give the "fattening" instability curve dividing instability from stability under radial perturbations. In Figure 4, we have only represented the set of parameters such that the spherical shells are not local 
minimizers for spherically symmetric perturbations, as well as all the parameters $(\gamma, \alpha)$ used for 3D numerical simulations in this article in Table 2. Just as we have observed in the 2D case, crossing the lower border of this set leads to a "fattening" instability of the spherical shell.

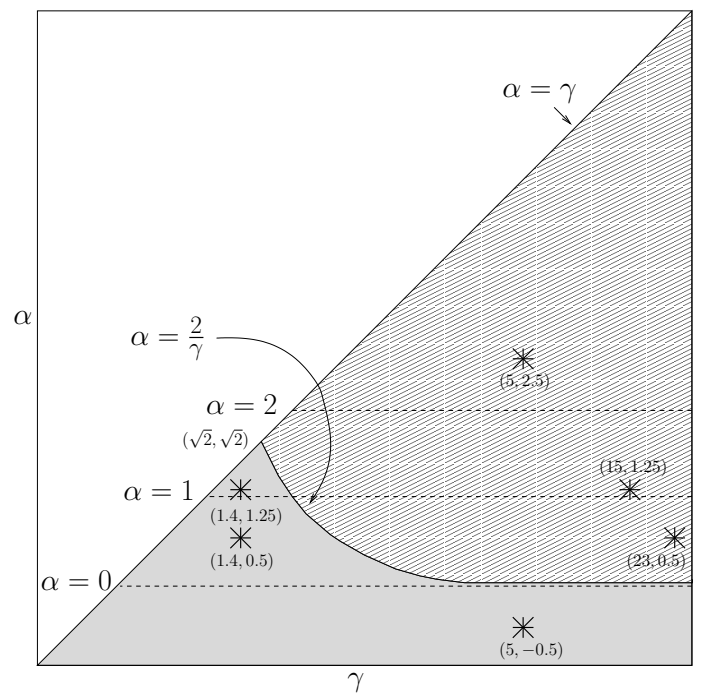

Figure 4. Sketch of all the computed cases in dimension $N=3$. The parameters $(\gamma, \alpha)$ used in Table 2 are marked with $*$. Notice that $\alpha<\gamma$ is necessary for the interaction potential to be confining. The curve is the limit between parameters leading to spherical shell solutions for radial perturbations (above the curve) and to minimizers of dimensionality 3 (below the curve).

Notice finally that it is also possible to modify the dimensionality of the local minimizers with other perturbations of power law potentials. As an example, in Table 4, we consider the following perturbations of the power law potential (5):

$$
W(x)=-\frac{|x|^{\alpha}}{\alpha}+\frac{|x|^{\gamma}}{\gamma}+\frac{3}{2 p} \cos (p x), \quad \alpha<\gamma, \quad p=3,5 .
$$

In Table 4 we have represented the power-law case in the first column, and the perturbations in the next two. For $(\gamma, \alpha)=(2,1.5)$, the unperturbed power-law potential leads to a local minimizer with Hausdorff dimension two. When we add the perturbation $p=3$, the dimension of the minimizer changes to one. Notice that the perturbation does not alter the local behavior of the potential at the origin or at infinity, suggesting that Theorem 1 is probably sharp at least in terms of natural dimensions.

Acknowledgments. We would like to thanks James von Brecht for multiple insightful conversation concerning the numerical section of this paper. DB and JAC were supported by the projects Ministerio de Ciencia e Innovación MTM2011-27739-C04-02 and 2009-SGR-345 from Agència de Gestió d'Ajuts Universitaris i de Recerca-Generalitat de Catalunya. JAC acknowledges support from the Royal Society through a Wolfson Research Merit Award. This work was supported by Engineering and Physical Sciences Research Council grant number EP/K008404/1. GR was supported by Award No. KUK-I1- 007-43 of Peter A. Markowich, made by King Abdullah University of Science and Technology (KAUST). DB, JAC and GR acknowledge partial support from CBDif-Fr ANR-08-BLAN-0333-01 project. TL acknowledges the support from NSF Grant DMS-1109805. 


\begin{tabular}{||l|l|l|l||}
\hline & Powers & $p=3$ & $p=5$ \\
\hline & & & \\
\hline
\end{tabular}

TABLE 4. Local minimizers with the power-law potential (5) and the perturbed potential (39), $n=10,000$.

\section{REFERENCES}

[1] L. Ambrosio, N. Gigli, and G. Savaré. Gradient flows in metric spaces and in the space of probability measures. Lectures in Mathematics ETH Zürich. Birkhäuser Verlag, 2008.

[2] Carrillo J. A. Balagué, D. and Y. Yao. Confinement for repulsive-attractive kernels. Preprint.

[3] D. Balagué, Carrillo, T. J. A., Laurent, and G. Raoul. Nonlocal interactions by repulsive-attractive potentials: radial ins/stability. preprint, 2011.

[4] A. J. Bernoff and C. M. Topaz. A primer of swarm equilibria. SIAM J. Appl. Dyn. Syst., 10(1):212-250, 2011.

[5] A. Bertozzi, J. A. Carrillo, and T. Laurent. Blowup in multidimensional aggregation equations with mildly singular interaction kernels. Nonlinearity, 22:683-710, 2009.

[6] A. Bertozzi, J. Garnett, and T. Laurent. Characterization of radially symmetric finite time blowup in multidimensional aggregation equations.

[7] A. Bertozzi and T. Laurent. Finite-time blow-up of solutions of an aggregation equation in $\mathbb{R}^{n}$. Comm. Math. Phys., 274:717-735, 2007.

[8] A. Bertozzi, T. Laurent, and J. Rosado. Lp theory for the multidimensional aggregation equation. Comm. Pure Appl. Math., 64(1):45-83, 2011.

[9] A. L. Bertozzi, T. Laurent, and F. Léger. Aggregation and spreading via the newtonian potential: the dynamics of patch solutions. Mathematical Models and Methods in Applied Sciences, 22(supp01):1140005, 2012.

[10] J. A. Carrillo, M. Di Francesco, A. Figalli, T. Laurent, and D. Slepčev. Confinement in nonlocal interaction equations. Nonlinear Anal., 75(2):550-558, 2012.

[11] J. A. Carrillo, M. Di Francesco, A. Figalli, T. Laurent, and D. Slepčev. Global-in-time weak measure solutions and finite-time aggregation for nonlocal interaction equations. Duke Math. J., 156:229-271, 2011.

[12] J. A. Carrillo, R. J. McCann, and C. Villani. Kinetic equilibration rates for granular media and related equations: entropy dissipation and mass transportation estimates. Rev. Mat. Iberoamericana, 19(3):971-1018, 2003.

[13] J. A. Carrillo, R. J. McCann, and C. Villani. Contractions in the 2-wasserstein length space and thermalization of granular media. Arch. Rat. Mech. Anal., 179:217-263, 2006.

[14] M. R. D'Orsogna, Y. Chuang, A. Bertozzi, and L. Chayes. Self-propelled particles with soft-core interactions: patterns, stability and collapse. Phys. Rev. Lett., 96(104302), 2006.

[15] J. P. K. Doye, D. J. Wales, and R. S. Berry. The effect of the range of the potential on the structures of clusters. J. Chem. Phys., 103:4234-4249, 1995.

[16] Kenneth Falconer. Fractal geometry. John Wiley \& Sons Inc., Hoboken, NJ, second edition, 2003. Mathematical foundations and applications.

[17] K. Fellner and G. Raoul. Stable stationary states of non-local interaction equations. Math. Models Methods Appl. Sci., 20(12):2267-2291, 2010.

[18] K. Fellner and G. Raoul. Stability of stationary states of non-local equations with singular interaction potentials. Math. Comput. Modelling, 53(7-8):1436-1450, 2011.

[19] R. C. Fetecau, Y. Huang, and T. Kolokolnikov. Swarm dynamics and equilibria for a nonlocal aggregation model. Nonlinearity, 24(10):2681-2716, 2011.

[20] Clark R. Givens and Rae Michael Shortt. A class of Wasserstein metrics for probability distributions. Michigan Math. J., 31(2):231-240, 1984.

[21] M. F. Hagan and D. Chandler. Dynamic pathways for viral capsid assembly. Biophysical Journal, 91:42-54, 2006. 
[22] T. Kolokolnikov, Y. Huang, and M. Pavlovski. Singular patterns for an aggregation model with a confining potential. to appear in Physica D.

[23] T. Kolokonikov, H. Sun, D. Uminsky, and A. Bertozzi. Stability of ring patterns arising from 2d particle interactions. Physical Review E, 84(1):015203, 2011.

[24] T. Laurent. Local and global existence for an aggregation equation. Communications in Partial Differential Equations, 32:1941-1964, 2007.

[25] R. J. McCann. Stable rotating binary stars and fluid in a tube. Houston J. Math., 32(2):603-631, 2006

[26] A. Mogilner and L. Edelstein-Keshet. A non-local model for a swarm. J. Math. Bio., 38:534-570, 1999.

[27] A. Mogilner, L. Edelstein-Keshet, L. Bent, and A. Spiros. Mutual interactions, potentials, and individual distance in a social aggregation. J. Math. Biol., 47(4):353-389, 2003.

[28] Papadimitrakis. Notes on classical potential theory. see link to web page, 2003.

[29] J. Pérez and A. Ros. Properly embedded minimal surfaces with finite total curvature. In The global theory of minimal surfaces in flat spaces (Martina Franca, 1999), volume 1775 of Lecture Notes in Math., pages 15-66. Springer, Berlin, 2002.

[30] G. Raoul. Non-local interaction equations: Stationary states and stability analysis. Differential Integral Equations, 25(5-6):417-440, 2012.

[31] M.C. Rechtsman, F.H. Stillinger, and S. Torquato. Optimized interactions for targeted self-assembly: application to a honeycomb lattice. Phys. Rev. Lett., 95(22), 2005.

[32] Hui Sun, David Uminsky, and Andrea L. Bertozzi. Stability and clustering of self-similar solutions of aggregation equations. J. Math. Phys., 53:115610, 2012.

[33] C. Topaz, A. Bernoff, S. S. Logan, and W. Toolson. A model for rolling swarms of locusts. Eur. Phys. J. Special Topics, 157:93-109, 2008.

[34] C. Villani. Topics in optimal transportation, volume 58 of Graduate Studies in Mathematics. American Mathematical Society, Providence, RI, 2003.

[35] J. von Brecht and D. Uminsky. On soccer balls and linearized inverse statistical mechanics. to appear in J. Nonlinear Sci.

[36] J. von Brecht, D. Uminsky, T. Kolokolnikov, and A. Bertozzi. Predicting pattern formation in particle interactions. Math. Mod. Meth. Appl. Sci., 22:1140002, 2012.

[37] D. J. Wales. Energy landscapes of clusters bound by short-ranged potentials. Chem. Eur. J. Chem. Phys., 11:2491-2494, 2010

1 Departament de Matemàtiques, Universitat Autònoma de Barcelona, E-08193 Bellaterra, Spain. E-MAIL: dbalague@mat.uab.cat.

2 Department of Mathematics, Imperial College London, South Kensington Campus, London SW7 2AZ, UK. E-MAIL: carrillo@imperial.ac.uk

3 Department of Mathematics, University of California - Riverside, Riverside, CA 92521, USA. E-MAIL: laurent@math.ucr.edu.

${ }^{4}$ Centre d'Ecologie Fonctionnelle et Evolutive, UMR 5175, CNRS,, 1919 Route de Mende, 34293 Montpellier Cedex 5, France. E-Mail: raoul@cefe.cnrs.fr. 